\title{
Existence and Uniqueness of the Solutions for Some Initial-Boundary Value Problems with the Fractional Dynamic Boundary Condition
}

\author{
Mykola Krasnoschok and Nataliya Vasylyeva \\ Institute of Applied Mathematics and Mechanics of NAS of Ukraine, R.Luksemburg Street 74, Donetsk 83114, Ukraine \\ Correspondence should be addressed to Nataliya Vasylyeva; vasylyeva@iamm.ac.donetsk.ua
}

Received 22 April 2013; Accepted 28 July 2013

Academic Editor: Antonin Novotny

Copyright (C) 2013 M. Krasnoschok and N. Vasylyeva. This is an open access article distributed under the Creative Commons Attribution License, which permits unrestricted use, distribution, and reproduction in any medium, provided the original work is properly cited.

\begin{abstract}
In this paper, we analyze some initial-boundary value problems for the subdiffusion equation with a fractional dynamic boundary condition in a one-dimensional bounded domain. First, we establish the unique solvability in the Hölder space of the initialboundary value problems for the equation $\partial_{t}^{\alpha} u(x, t)=L u(x, t)+f_{0}(x, t), \alpha \in(0,1)$, where $L$ is a uniformly elliptic operator with smooth coefficients with the fractional dynamic boundary condition. Second, we apply the contraction theorem to prove the existence and uniqueness locally in time in the Hölder classes of the solution to the corresponding nonlinear problems.
\end{abstract}

\section{Introduction}

Let $a$ and $b$ be any numbers from $R^{1}$ and let $\Omega=(a, b), \Omega_{T}=$ $\Omega \times(0, T) ; \Gamma_{1 T}=\{a\} \times[0, T] ; \Gamma_{2 T}=\{b\} \times[0, T] ; T>0$ be a fixed value. In this paper, we consider a partial differential equation with the fractional derivative in time $t$ as follows:

$$
\begin{aligned}
\partial_{t}^{\alpha} u(x, t)-a_{0}(x, t) \frac{\partial^{2} u(x, t)}{\partial x^{2}}+a_{1}(x, t) \frac{\partial u(x, t)}{\partial x} \\
+a_{2}(x, t) u(x, t)=f_{0}(x, t), \\
\quad(x, t) \in \Omega_{T}, \alpha \in(0,1] .
\end{aligned}
$$

Here, $\partial_{t}^{\alpha}$ denotes the Caputo fractional derivative with respect to $t$ and is defined by (see, e.g., (2.4.6) in [1]),

$$
\partial_{t}^{\alpha} g=\frac{1}{\Gamma(1-\alpha)} \frac{d}{d t} \int_{0}^{t} \frac{g(\tau) d \tau}{(t-\tau)^{\alpha}}-\frac{g(0)}{\Gamma(1-\alpha) t^{\alpha}}, \quad \alpha \in(0,1),
$$

where $\Gamma$ is the gamma function, $a_{i}(x, t), i=\overline{0,2}$, are the given functions, and $a_{0}(x, t)$ is a positive. Note that if $\alpha=1$, then (1) represents a parabolic equation. As we are interested in the fractional cases, we restrict the order $\alpha$ to the case $0<\alpha<1$.

We will solve (1) satisfying the following conditions:

$$
u(x, 0)=u_{0}(x) \text { in } \Omega \text { (initial condition), }
$$

the fractional dynamic boundary condition on $\Gamma_{2 T}$ :

$$
\partial_{t}^{\alpha} u(x, t)+b_{0}(t) \frac{\partial u(x, t)}{\partial x}+b_{1}(t) u(x, t)=f_{1}(t) \quad \text { on } \Gamma_{2 T}
$$

and one of the following conditions on $\Gamma_{1 T}$ : the Dirichlet boundary condition:

$$
u(x, t)=f_{2}(t) \quad \text { on } \Gamma_{1 T},
$$

or the Neumann boundary condition:

$$
\frac{\partial u(x, t)}{\partial x}=f_{3}(t) \quad \text { on } \Gamma_{1 T},
$$

or the fractional dynamic boundary condition:

$$
\partial_{t}^{\alpha} u(x, t)-b_{2}(t) \frac{\partial u(x, t)}{\partial x}+b_{3}(t) u(x, t)=f_{4}(t) \quad \text { on } \Gamma_{1 T} .
$$

Here, $b_{0}(t)$ and $b_{2}(t)$ are given positive functions, and $b_{1}(t), b_{3}(t)$, and $f_{i}(t), i=\overline{2,4}$, are given functions.

Concerning problems (1)-(6), they have the following features. First of all, (4) is a fractional dynamic boundary 
condition; next, these problems are formulated for the subdiffusion linear equation.

Note that if $\alpha=1$, conditions (4) and (6) are called normal dynamic boundary conditions. These conditions are very natural in many mathematical models, including heat transfer in a solid in contact with a moving fluid, thermoelasticity, diffusion phenomena, and problems in fluid dynamics, and in the Stefan problem, (see [2-4] and the references therein). At the present moment, there are a lot of works concerning linear and nonlinear problems with dynamic boundary conditions. Here we make no pretence to provide a complete survey on the results related to problems of the type (1)-(6), if $\alpha=1$, and present only some of them. The initial-boundary value problems for the heat equation in the certain shape of domains with linear dynamic boundary condition have been solved with the separation variables method or with the Laplace transformation in [3]. In the case of smooth domains, these problems have been researched with the approaches of the general theory for evolution equations in Hilbert and Banach spaces, and the weak solutions of the above mentioned problems have been obtained in [5-7]. Using the Schauder method, Grigor'eva and Mogilevskii [8] have got the coercive estimates of the solution in the anisotropic Sobolev spaces. The one-to-one solvability in the case of the linear parabolic equation with variable coefficients has been proved by Bazaliy [4] in the Hölder spaces and by Bizhanova and Solonnikov [9] in the weighted Hölder classes. The global and local existence for the solution to initial-boundary value problem for linear and quasilinear equations with nonlinear dynamic boundary conditions has been discussed in [10-12] (see also references there).

Over the past few decades, an intensive effort has been put into developing theoretical models for systems with diffusive motion that cannot be modelled as the standard Brownian motion $[13,14]$. The signature of this anomalous diffusion is that the mean square displacement of the diffusing species $\left\langle(\Delta \mathbf{x})^{2}\right\rangle$ scales as a nonlinear power law in time, that is, $\left\langle(\Delta \mathbf{x})^{2}\right\rangle \sim t^{\alpha}$. If $\alpha \in(0,1)$, this is referred to as subdiffusion. In recent years, the additional motivation for these studies has been stimulated by experimental measurements of subdiffusion in porous media [15], glass forming materials [16], and biological media [17]. The review paper by Klafter et al. [18] provides numerous references to physical phenomena in which anomalous diffusion occurs.

Here we refer to several works on the mathematical treatments for linear equation (1). Kochubei [19, 20], and Pskhu $[21,22]$ constructed the fundamental solution in $R^{d}$ and proved the maximum principle for the Cauchy problem. Gejji and Jafari [23] solved a nonhomogeneous fractional diffusion-wave equation in a one-dimensional bounded domain. Metzler and Klafter [14], using the method of images and the Fourier-Laplace transformation technique, obtained the solutions of different boundary value problems for the homogenous fractional diffusion equation in a half-space and in a box. Agrawal [24] constructed a solution of a fractional diffusion equation using a finite transform technique and presented numerical results in a one-dimensional bounded domain. Mophou and N'Guérékata [25] and Sakamoto and
Yamamoto [26] proved the one-valued solvability of the initial-boundary value problem for the fractional diffusion equation with variable coefficients which is $t$-independent with the homogenous Dirichlet conditions in the Sobolev space. Note that, in [26], the authors obtained the certain regularities of the solution given by the eigenfunction expansions and established several results of uniqueness for related inverse problems.

As source books related with fractional derivatives, see the work of Samko et al. [27] which is an encyclopedic treatment of the fractional calculus and also Kilbas et al. [1], Mainardi [28], Podlubny [29], and Pskhu [21].

As for the quasilinear equation like (1), Clément et al. [30] analyzed the abstract fractional parabolic quasilinear equations. Via maximal regularity results in the corresponding linear equation, they arrived to results on existence (locally in time), uniqueness, and continuation on the quasilinear equation in the BUC classes with a weight. As for investigation of the problem with fractional dynamic boundary conditions, Kirane and Tatar [31] have analyzed the issue of nonexistence of local and global solutions for elliptic systems with nonlinear fractional dynamic boundary conditions.

To the authors' best knowledge, there are no works published concerning the solvability of problems (1)-(6) in the Hölder classes. The first purpose of this paper is to prove the well-posedness and the regularity of the solutions to problems (1)-(6) in the smooth classes. Second, we obtain a local in time solvability in the smooth classes of the corresponding nonlinear problems. This paper is organized as follows. In the second section, we state the main results, Theorems 3-5, and define the functional spaces. In Section 3, we establish the one-valued solvability of certain model problems in $R_{T}^{+}$. The principal results of this section are given in Theorems 9 and 13. In Section 4, we prove the main results of this paper. To this end, we will combine ideas from [32] with coercive estimates of the solutions to the corresponding model problems (Section 3). In Section 5, we address the corresponding nonlinear problems. We first reduce them to a form $\mathfrak{A} w=\mathfrak{I}(w)$, where $\mathfrak{I}(w)$ is a nonlinear function of $w$ and $\mathfrak{A}$ is the linear operator derived in Section 4; that is, $\mathfrak{A}^{-1} \mathfrak{T}$ is the solution of the model problem for data $\mathfrak{T}$. Setting $\mathfrak{S}=\mathfrak{A}^{-1} \mathfrak{T}$, we will then prove that the mapping $w \rightarrow \mathfrak{S}(w)$, where $\mathfrak{S}(w)=\mathfrak{A}^{-1} \mathfrak{T} w$, is a contraction, so that it has a unique fixed point. The principal results of this section are formulated in Theorem 18 and Remarks 19 and 20. The Appendix contains the proofs of some auxiliary assertions which are applied in Section 3.

\section{The Functional Spaces and the Main Results}

Let us introduce the functional spaces. Let $\Omega$ be a bounded or an unbounded domain in $R^{1}, \bar{x}, x \in \bar{\Omega} ; t, \tau \in[0, T] ; \theta, \gamma \in$ $(0,1)$. Denote

$$
\begin{aligned}
\langle v\rangle_{x, \Omega_{T}}^{(\theta)} & =\sup _{(x, t),(\bar{x}, t) \in \Omega_{T}, x \neq \bar{x}} \frac{|v(x, t)-v(\bar{x}, t)|}{|x-\bar{x}|^{\theta}} ; \\
\langle v\rangle_{t, \Omega_{T}}^{(\gamma)} & =\sup _{(x, t),(x, \tau) \in \Omega_{T}, t \neq \tau} \frac{|v(x, t)-v(x, \tau)|}{|t-\tau|^{\gamma}} .
\end{aligned}
$$


Definition 1. We will say that functions $v(x, t) \in C^{\theta,(\theta / 2) \alpha}\left(\bar{\Omega}_{T}\right)$ and that $w(x, t) \in C^{2+\theta,((2+\theta) / 2) \alpha}\left(\bar{\Omega}_{T}\right)$ if and only if the functions $v(x, t)$ and $\partial^{k} w(x, t) / \partial x^{k}, k=\overline{0,2}, \partial_{t}^{\alpha} w(x, t)$ are continuous and the following norms are finite:

$$
\begin{aligned}
& \|v\|_{C^{\theta,(\theta / 2) \alpha}\left(\bar{\Omega}_{T}\right)}=\sup _{\bar{\Omega}_{T}}|v(x, t)|+\langle v\rangle_{x, \Omega_{T}}^{(\theta)}+\langle v\rangle_{t, \Omega_{T}}^{((\theta / 2) \alpha)}, \\
& \|w\|_{C^{2+\theta,((2+\theta) / 2) \alpha}\left(\bar{\Omega}_{T}\right)} \\
& =\sum_{k=0}^{2} \sup _{T}\left|\frac{\partial^{k} w}{\partial x^{k}}\right|+\sup _{\bar{\Omega}_{T}}\left|\partial_{t}^{\alpha} w\right|+\left\langle\frac{\partial^{2} w}{\partial x^{2}}\right\rangle_{x, \Omega_{T}}^{(\theta)} \\
& +\left\langle\partial_{t}^{\alpha} w\right\rangle_{x, \Omega_{T}}^{(\theta)}+\sum_{k=1}^{2}\left\langle\frac{\partial^{k} w}{\partial x^{k}}\right\rangle_{t, \Omega_{T}}^{(((2+\theta-k) / 2) \alpha)} \\
& +\left\langle\partial_{t}^{\alpha} w\right\rangle_{t, \Omega_{T}}^{((\theta / 2) \alpha)} .
\end{aligned}
$$

Note that if $\alpha=1$, the spaces $C^{l+\theta,((l+\theta) / 2) \alpha}\left(\bar{\Omega}_{T}\right), l=0,2$, coincide with the ordinary Hölder spaces (see (1.10)-(1.12) in Chapter 1 in [32]). Further, we also use the Banach spaces $C^{\gamma \alpha}([0, T])$ and $C^{(1+\gamma) \alpha}([0, T])$ of the functions $V(t)$ and $W(t)$ with the finite norms

$$
\begin{gathered}
\|V\|_{C^{\gamma \alpha}([0, T])}=\sup _{[0, T]}|V(t)|+\langle V\rangle_{t,[0, T]}^{(\gamma \alpha)}, \\
\|W\|_{C^{(1+\gamma) \alpha}([0, T])}=\sup _{[0, T]}|W(t)|+\sup _{[0, T]}\left|\partial_{t}^{\alpha} W\right| \\
\\
+\left\langle\partial_{t}^{\alpha} W\right\rangle_{t,[0, T]}^{(\gamma \alpha)} .
\end{gathered}
$$

In a similar way, we introduce the spaces $C^{(k+\gamma) \alpha}\left(\partial \Omega_{T}\right), k=$ 0,1 .

Definition 2. We will say that functions $v_{1}(x, t) \in$ $C_{0}^{\theta,(\theta / 2) \alpha}\left(\bar{\Omega}_{T}\right)$ and that $v_{2}(x, t) \in C_{0}^{2+\theta,((2+\theta) / 2) \alpha}\left(\bar{\Omega}_{T}\right)$ if and only if $v_{1}(x, t) \in C^{\theta,(\theta / 2) \alpha}\left(\bar{\Omega}_{T}\right)$ and $v_{1}(x, 0)=0$ and $v_{2}(x, t) \in C^{2+\theta,((2+\theta) / 2) \alpha}\left(\bar{\Omega}_{T}\right)$ and $v_{2}(x, 0)=\partial_{t}^{\alpha} v_{2}(x, 0)=0$.

If $u_{1}(t) \in C^{\gamma \alpha}([0, T]), u_{1}(0)=0\left(u_{2}(t) \in C^{(1+\gamma) \alpha}([0, T])\right.$, and $\left.u_{2}(0)=\partial_{t}^{\alpha} u_{2}(0)=0\right)$, then $u_{1}(t) \in C_{0}^{\gamma \alpha}([0, T])\left(u_{2}(t) \in\right.$ $\left.C_{0}^{(1+\gamma) \alpha}([0, T])\right)$.

We introduce the spaces of $C_{0}^{(k+\gamma) \alpha}\left(\partial \Omega_{T}\right), k=0,1$, with the same way.

Let $\boldsymbol{\delta}_{1}$ and $\boldsymbol{\delta}_{2}$ be some positive numbers. We assume that the given functions $a_{i}(x, t), i=\overline{0,2}$, and $b_{j}(t), j=\overline{0,2}$, in (1), (4), and (7) are subject to the following conditions:

(i) $a_{0}(x, t) \geq \boldsymbol{\delta}_{1}>0 \quad$ in $\bar{\Omega}_{T} ; \quad b_{0}(t), b_{2}(t) \geq \boldsymbol{\delta}_{2}>0$

$$
\forall t \in[0, T] \text {; }
$$

(ii) $a_{i}(x, t) \in C^{\theta,(\theta \alpha / 2)}\left(\bar{\Omega}_{T}\right), \quad b_{j}(t) \in C^{((1+\theta) / 2) \alpha}([0, T])$,

$$
i=\overline{0,2}, \quad j=\overline{0,3} \text {; }
$$

$$
\text { (iii) } \begin{aligned}
f_{1}(0) & -b_{0}(0) \frac{\partial u_{0}(b)}{\partial x}-b_{1}(0) u_{0}(b) \\
= & a_{0}(b, 0) \frac{\partial^{2} u_{0}(b)}{\partial x^{2}}-a_{1}(b, 0) \frac{\partial u_{0}(b)}{\partial x} \\
& -a_{2}(b, 0) u_{0}(b)+f_{0}(b, 0) ;
\end{aligned}
$$

and one of the following:

$$
\begin{gathered}
f_{2}(0)=u_{0}(a), \\
\partial_{t}^{\alpha} f_{2}(0)=a_{0}(a, 0) \frac{\partial^{2} u_{0}(a)}{\partial x^{2}}-a_{1}(a, 0) \frac{\partial u_{0}(a)}{\partial x} \\
-a_{2}(a, 0) u_{0}(a)+f_{0}(a, 0)
\end{gathered}
$$

(in the case of Dirichlet condition (5));

or

$$
f_{3}(0)=\frac{\partial u_{0}(a)}{\partial x}
$$

(in the case of Neumann condition (6));

or

$$
\begin{aligned}
f_{4}(0) & +b_{2}(0) \frac{\partial u_{0}(a)}{\partial x}-b_{3}(0) u_{0}(a) \\
= & a_{0}(a, 0) \frac{\partial^{2} u_{0}(a)}{\partial x^{2}}-a_{1}(a, 0) \frac{\partial u_{0}(a)}{\partial x} \\
& -a_{2}(a, 0) u_{0}(a)+f_{0}(a, 0)
\end{aligned}
$$

(in the case of fractional dynamic boundary condition (7)) .

Note that requirements (15)-(18) are called the consistency conditions.

The main results of our paper are the following:

Theorem 3. Let $\theta, \alpha \in(0,1)$, and conditions (13)-(16) hold, and $f_{0}(x, t) \in C^{\theta,(\theta / 2) \alpha}\left(\bar{\Omega}_{T}\right), u_{0}(x) \in C^{2+\theta}(\bar{\Omega}), f_{1}(t) \in$ $C^{((1+\theta) / 2) \alpha}([0, T]), f_{2}(t) \in C^{((2+\theta) / 2) \alpha}([0, T])$ for any positive number $T$. Then there exists a unique solution $u(x, t)$ of problem (1)-(5): $u(x, t) \in C^{2+\theta,((2+\theta) / 2) \alpha}\left(\bar{\Omega}_{T}\right), \partial_{t}^{\alpha} u(x, t) \in$ $C^{((1+\theta) / 2) \alpha}\left(\Gamma_{2 T}\right)$, and

$$
\begin{aligned}
\|u\|_{C^{2+\theta,((2+\theta) / 2) \alpha}\left(\bar{\Omega}_{T}\right)}+\left\|\partial_{t}^{\alpha} u\right\|_{C^{((1+\theta) / 2) \alpha}\left(\Gamma_{2 T}\right)} & \\
\leq C & {\left[\left\|f_{0}\right\|_{C^{\theta,(\theta / 2) \alpha}\left(\bar{\Omega}_{T}\right)}+\left\|u_{0}\right\|_{C^{2+\theta}(\bar{\Omega})}\right.} \\
& \left.\quad\left\|f_{1}\right\|_{C^{(1+\theta) / 2) \alpha}([0, T])}+\left\|f_{2}\right\|_{C^{(2+\theta) / 2) \alpha}([0, T])}\right],
\end{aligned}
$$

where a positive constant $C$ depends only on the measure of $\Omega$ and $\left\|a_{i}\right\|_{C^{\theta,(\theta / 2) \alpha}\left(\bar{\Omega}_{T}\right)}, i=\overline{0,2},\left\|b_{j}\right\|_{C^{(1+\theta) / 2) \alpha}([0, T])}, j=0,1$. 
Theorem 4. Let $\theta, \alpha \in(0,1)$, and conditions (13)-(15), and (17) hold, and let $f_{0}(x, t) \in C^{\theta,(\theta / 2) \alpha}\left(\bar{\Omega}_{T}\right), u_{0}(x) \in C^{2+\theta}(\bar{\Omega})$, $f_{i}(t) \in C^{((1+\theta) / 2) \alpha}([0, T]), i=1,3$ for any positive number $T$. Then there exists a unique solution $u(x, t)$ of problem (1)(4), (6) as follows: $u(x, t) \in C^{2+\theta,((2+\theta) / 2) \alpha}\left(\bar{\Omega}_{T}\right), \partial_{t}^{\alpha} u(x, t) \in$ $C^{((1+\theta) / 2) \alpha}\left(\Gamma_{2 T}\right)$, and

$$
\begin{aligned}
& \|u\|_{C^{2+\theta,((2+\theta) / 2) \alpha}\left(\bar{\Omega}_{T}\right)}+\left\|\partial_{t}^{\alpha} u\right\|_{C^{(1+\theta) / 2) \alpha}\left(\Gamma_{2 T}\right)} \\
& \leq C\left[\left\|f_{0}\right\|_{C^{\theta,(\theta / 2) \alpha}\left(\bar{\Omega}_{T}\right)}+\left\|u_{0}\right\|_{C^{2+\theta}(\bar{\Omega})}\right. \\
& \left.+\left\|f_{1}\right\|_{C^{((1+\theta) / 2) \alpha}([0, T])}+\left\|f_{3}\right\|_{C^{((1+\theta) / 2) \alpha}([0, T])}\right],
\end{aligned}
$$

where $C$ is a positive constant and depends only on $\left\|b_{j}\right\|_{C^{((1+\theta) / 2) \alpha}([0, T])}, j=0,1,\left\|a_{i}\right\|_{C^{\theta,(\theta / 2) \alpha}\left(\bar{\Omega}_{T}\right)}, i=\overline{0,2}$, and the measure of $\Omega$.

Theorem 5. Let $\theta, \alpha \in(0,1)$, and conditions (13)-(15) and (18) hold, and $f_{0}(x, t) \in C^{\theta,(\theta / 2) \alpha}\left(\bar{\Omega}_{T}\right), u_{0}(x) \in C^{2+\theta}(\bar{\Omega})$, $f_{i}(t) \in C^{((1+\theta) / 2) \alpha}([0, T])$, and $i=1,4$ for any positive number $T$. Then there exists a unique solution $u(x, t)$ of problem (1)(4), (7) as follows: $u(x, t) \in C^{2+\theta,((2+\theta) / 2) \alpha}\left(\bar{\Omega}_{T}\right), \partial_{t}^{\alpha} u(x, t) \in$ $C^{((1+\theta) / 2) \alpha}\left(\Gamma_{i T}\right), i=1,2$, and

$$
\begin{gathered}
\|u\|_{C^{2+\theta,((2+\theta) / 2) \alpha}\left(\bar{\Omega}_{T}\right)}+\sum_{i=1}^{2}\left\|\partial_{t}^{\alpha} u\right\|_{C^{((1+\theta) / 2) \alpha}\left(\Gamma_{i T}\right)} \\
\leq C\left[\left\|f_{0}\right\|_{C^{\theta,(\theta / 2) \alpha}\left(\bar{\Omega}_{T}\right)}+\left\|u_{0}\right\|_{C^{2+\theta}(\bar{\Omega})}\right. \\
\left.+\left\|f_{1}\right\|_{C^{((1+\theta) / 2) \alpha}([0, T])}+\left\|f_{4}\right\|_{C^{((1+\theta) / 2) \alpha}([0, T])}\right],
\end{gathered}
$$

where $C$ is a positive constant and depends only on $\left\|b_{j}\right\|_{C^{((1+\theta) / 2) \alpha}([0, T])}, j=\overline{0,3},\left\|a_{i}\right\|_{C^{\theta,(\theta / 2) \alpha}\left(\bar{\Omega}_{T}\right)}, i=\overline{0,2}$, and the measure of $\Omega$.

\section{Model Problems}

Let $D^{+}=(0,+\infty)$, and $D_{T}^{+}=D^{+} \times(0, T)$, and $a_{0}$ and $b_{0}$ be some positive numbers. Here we will discuss the first initial-boundary value problem for the fractional diffusion equation in $D_{T}^{+}$and the initial-boundary value problem with the fractional boundary condition in $D_{T}^{+}$.

3.1. The Solvability of the First Initial-Boundary Value Problem for the Subdiffusion Equation. We look for the function $v(x, t)$ by the following conditions:

$$
\begin{gathered}
\partial_{t}^{\alpha} v(x, t)-a_{0} \frac{\partial^{2} v(x, t)}{\partial x^{2}}=g_{0}(x, t) \quad \text { in } D_{T}^{+}, \alpha \in(0,1) \\
v(x, 0)=v_{0}(x), \quad x \in D^{+} ; \\
v(x, t) \longrightarrow 0 \quad \text { if } x \longrightarrow+\infty, \forall t \in[0, T] \\
v(0, t)=g_{1}(t), \quad t \in[0, T]
\end{gathered}
$$

where $g_{0}(x, t)$ and $g_{1}(t), v_{0}(x)$ are some given functions.
We assume that the following conditions hold:

(i) $v_{0}(0)=g_{1}(0) ; \quad \partial_{t}^{\alpha} g_{1}(0)=a_{0} \frac{\partial^{2} v_{0}}{\partial x^{2}}+g_{0}(x, 0)$;

(ii) $v_{0}(x), g_{0}(x, t) \equiv 0$, if $|x|<R_{0}, \forall t \in[0, T]$,

for some positive number $R_{0}$.

First, we will study problem (22)-(24) under restriction

$$
v_{0}(x), g_{0}(x, t) \equiv 0 .
$$

We will search a solution of (22)-(24) under restriction (27) in the class $C^{2+\theta,((2+\theta) / 2) \alpha}\left(\bar{D}_{T}^{+}\right)$, if $g_{1}(t) \in C^{((2+\theta) / 2) \alpha}([0, T])$ for $\theta \in(0,1)$.

Note that conditions (25) and (26) together with restriction (27) allow us to apply the Laplace transformation in $t$ to the right hand sides of (22)-(24). Indeed, conditions (25)-(27) mean that the right hand sides in (22)-(24) except $g_{1}(t)$ equal zero and $g_{1}(0)=0$. Thus, we can extend the right hand sides in (22)-(24) by 0 for $t \leq 0$ and save, for simplicity, the same notation for the extension of the function $g_{1}(t)$. Therefore, we can apply, at least formally, the Laplace transformation in $t$ to (22)-(24) in the case of (25)-(27) hold.

Denote by $\widehat{w}(x, p)$ the Laplace transformation of the function $w(x, t)$; that is,

$$
\widehat{w}(x, p)=\int_{0}^{+\infty} e^{-p t} w(x, t) d t .
$$

The Laplace transformation in (22)-(24) leads to the problem

$$
\begin{gathered}
p^{\alpha} \widehat{v}(x, p)-a_{0} \frac{\partial^{2} \widehat{v}(x, p)}{\partial x^{2}}=0, \quad x \in D^{+} ; \\
\widehat{v}(x, p) \longrightarrow 0 \quad \text { if } x \longrightarrow+\infty ; \\
\widehat{v}(0, p)=\widehat{g}_{1}(p) .
\end{gathered}
$$

Here we used the following formula from [33]:

$$
\widehat{\partial_{t}^{\alpha} w}=p^{\alpha} \widehat{w}(x, p)-p^{\alpha-1} w(x, 0) .
$$

One can easily check that the following function solves the equations in (29):

$$
\widehat{v}(x, p)=\exp \left(-\frac{x p^{\alpha / 2}}{\sqrt{a_{0}}}\right) g_{1}(p) .
$$

Due to formula (2.30) in [34] and the inverse Laplace transformation, we get the integral representations of $v(x, t)$ as follows:

$$
v(x, t)=\int_{0}^{t} G(x, t-\tau) g_{1}(\tau) d \tau,
$$

where

$$
G(x, t)=t^{-1} W\left(-\frac{x t^{-\alpha / 2}}{\sqrt{a_{0}}} ;-\frac{\alpha}{2}, 0\right) .
$$


Here $W(z ; \beta, \gamma)$ is the Wright function, which is defined for $z, \beta, \gamma \in \mathbb{C}$ as (see formula (1.8.1(27)) in v.3 [35])

$$
W(z ; \beta, \gamma)=\sum_{k=0}^{\infty} \frac{z^{k}}{k ! \Gamma(\beta k+\gamma)} .
$$

The main properties of the Wright functions are described in Chapters 4.1, v.1 and 18.1 v3 in [35], Chapter 1.11 in [1], Chapter 1.3 in [34], and Chapter 2 in $[21,36]$.

In Lemma 6, we describe the properties of the kernel $G(x, t)$ which will be necessary to estimate the function $v(x, t)$. Its proof is represented in Appendix A.

Lemma 6. Let $\kappa, \delta$, and $\bar{\theta}$ be some positive constants, $\kappa=\kappa(\alpha)$, $\bar{\theta} \in(0,1], x \in D^{+}, t \in[0, T]$. Then one has the following.

(i) The kernel $G(x, t)$ is positive for $x>0$.

$$
\text { (ii) } \int_{0}^{+\infty} G(x, t) d t=1 \text {. }
$$

(iii) $t G(x, t) \leq C \exp \left(-\kappa\left(x t^{-\alpha / 2}\right)^{1 /(1-\alpha / 2)}\right)$;

$$
\begin{gathered}
\left|\frac{\partial G(x, t)}{\partial t}\right| \leq C \frac{t^{-2}}{1+\left(x t^{-\alpha / 2}\right)^{4 / \alpha}} \\
\left|\frac{\partial G(x, t)}{\partial x}\right| \leq C \frac{t^{-1-\alpha / 2}}{1+\left(x t^{-\alpha / 2}\right)^{1+2 / \alpha}} .
\end{gathered}
$$$$
\text { (iv) } \int_{0}^{t} G(x, \tau) \tau^{(\bar{\theta} / 2) \alpha} d \tau \leq C t^{(\bar{\theta} / 2) \alpha} \text {. }
$$

(v) $\int_{\delta}^{+\infty}\left|\frac{\partial G(x, \tau)}{\partial x}\right| \tau^{(\bar{\theta} / 2) \alpha} d \tau \leq C \delta^{-(1-\bar{\theta}) \alpha / 2}$.

(vi) $\int_{\delta}^{+\infty}\left|\frac{\partial G(x, \tau)}{\partial \tau}\right| \tau^{\bar{\theta} \alpha / 2} d \tau \leq C \delta^{-1+\bar{\theta} \alpha / 2}$.

(vii) $\int_{0}^{t}(t-\tau)^{-\alpha} G(x, \tau) d \tau$

$$
\leq C t^{-\alpha} \exp \left(-\kappa\left(x t^{-(\alpha / 2)}\right)^{1 /(1-\alpha / 2)}\right) .
$$

(viii) $D_{t}^{\alpha} G(x, t)=a_{0} \frac{\partial^{2} G(x, t)}{\partial x^{2}} \quad$ in $D_{T}^{+}$,

where $D_{t}^{\alpha}$ is the Riemann-Liouville fractional derivative, and its definition is in (2.1.8) in [1].

Lemma 7. Let $\theta \in(0,1)$, and conditions (25)-(27) hold, and $g_{1}(t) \in C^{((2+\theta) / 2) \alpha}([0, T])$. Then the function $v(x, t)$ represented by formula (32) satisfies the following inequalities:

$$
\begin{gathered}
\sup _{\bar{D}_{T}^{+}}\left|\partial_{t}^{\alpha} v\right| \leq C\left(\sup _{[0, T]}\left|\partial_{t}^{\alpha} g_{1}\right|+\left\langle\partial_{t}^{\alpha} g_{1}\right\rangle_{t,[0, T]}^{((\theta / 2) \alpha)}\right) ; \\
\left\langle\partial_{t}^{\alpha} v\right\rangle_{t, D_{T}^{+}}^{((\theta / 2) \alpha)} \leq C\left\|\partial_{t}^{\alpha} g_{1}\right\|_{C^{(\theta / 2) \alpha}([0, T])} ; \\
\left\langle\partial_{t}^{\alpha} v\right\rangle_{x, D_{T}^{+}}^{(\theta)} \leq C\left\|\partial_{t}^{\alpha} g_{1}\right\|_{C^{(\theta / 2) \alpha}([0, T])} .
\end{gathered}
$$

Proof. First, we obtain the representation of $\partial_{t}^{\alpha} v$. To this end, we need the following properties of the fractional derivative (see Lemma 2.10 and formula (2.4.10) in [1]):

(i)

$$
\partial_{t}^{\alpha} \rho(t)=D_{t}^{\alpha} \rho(t), \quad \text { if } \rho(0)=0,
$$

where (see (2.1.8) in [1])

$D_{t}^{\alpha} \rho(t)=\frac{1}{\Gamma(1-\alpha)} \frac{d}{d t} \int_{0}^{t} \frac{\rho(\tau)}{(t-\tau)^{\alpha}} d \tau, \quad \alpha \in(0,1)$.

(ii) If the functions $\rho_{i}(t), i=1,2$, and $D_{t}^{\alpha} \rho_{1}(t)$ are bounded in $[0, T]$, then

$$
\begin{aligned}
& D_{t}^{\alpha} \int_{0}^{t} \rho_{1}(t-\tau) \rho_{2}(\tau) d \tau \\
& \quad=\int_{0}^{t} \rho_{2}(t-\tau) D_{\tau}^{\alpha} \rho_{1}(\tau) d \tau+\rho_{2}(t) \lim _{z \rightarrow 0} I_{z}^{1-\alpha} \rho_{1}(z),
\end{aligned}
$$$$
\forall t \in[0, T],
$$

where $I_{t}^{v}$ is the Riemann-Liouville fractional integral of order $v$ (see, e.g. (2.1.1) in [1])

$$
I_{t}^{\nu} \rho(t)=\frac{1}{\Gamma(\nu)} \int_{0}^{t} \frac{\rho(\tau)}{(t-\tau)^{1-\nu}} d \tau, \quad \operatorname{Re} \nu>0 .
$$

One can easily see that $\partial_{t}^{\alpha} g_{1}(t) \in C^{(\theta / 2) \alpha}([0, T])$ and $\partial_{t}^{\alpha} g_{1}(0)=0$ (see (25) and (27)). Then, using properties (46) and (48) and equality (36), we represent the function $\partial_{t}^{\alpha} v(x, t)$ as

$$
\begin{aligned}
& \partial_{t}^{\alpha} v(x, t) \\
& \quad=\int_{0}^{t} G(x, t-\tau)\left[\partial_{t}^{\alpha} g_{1}(\tau)-\partial_{t}^{\alpha} g_{1}(t)\right] d \tau+\partial_{t}^{\alpha} g_{1}(t) .
\end{aligned}
$$

Namely, this representation will be useful below. To prove inequality (43), we will use this representation, statement (i) from Lemma 6 and the first estimate in (37) as follows:

$$
\begin{aligned}
\left|\partial_{t}^{\alpha} v\right| \leq & \left\langle\partial_{t}^{\alpha} g_{1}\right\rangle_{t,[0, T]}^{((\theta / 2) \alpha)} \\
& \times \int_{0}^{t} \tau^{(\theta / 2) \alpha-1} \exp \left(-\kappa\left(x \tau^{-\alpha / 2}\right)^{(1 /(1-\alpha / 2))}\right) d \tau \\
& +\max _{[0, T]}\left|\partial_{t}^{\alpha} g_{1}\right| \\
\leq & C\left\|\partial_{t}^{\alpha} g_{1}\right\|_{C^{(\theta / 2) \alpha}([0, T])} .
\end{aligned}
$$

In view of $\partial_{t}^{\alpha} g_{1}(t)=0$, if $t \leq 0$, we can rewrite representation (50) as

$$
\begin{aligned}
& \partial_{t}^{\alpha} v(x, t) \\
& \quad=\int_{-\infty}^{t} G(x, t-\tau)\left[\partial_{t}^{\alpha} g_{1}(\tau)-\partial_{t}^{\alpha} g_{1}(t)\right] d \tau+\partial_{t}^{\alpha} g_{1}(t) .
\end{aligned}
$$


Let $x_{1}, x_{2} \in D^{+}$and $x_{2}>x_{1}$. Denote

$$
\Delta x:=x_{2}-x_{1}, \quad \Delta_{x} \partial_{t}^{\alpha} v:=\partial_{t}^{\alpha} v\left(x_{2}, t\right)-\partial_{t}^{\alpha} v\left(x_{1}, t\right) .
$$

Then,

$$
\begin{aligned}
\Delta_{x} \partial_{t}^{\alpha} v= & \int_{0}^{(\Delta x)^{2 / \alpha}} G\left(x_{2}, \tau\right)\left[\partial_{\tau}^{\alpha} g_{1}(t-\tau)-\partial_{\tau}^{\alpha} g_{1}(t)\right] d \tau \\
& +\int_{0}^{(\Delta x)^{2 / \alpha}} G\left(x_{1}, \tau\right)\left[\partial_{\tau}^{\alpha} g_{1}(t)-\partial_{\tau}^{\alpha} g_{1}(t-\tau)\right] d \tau \\
& +\int_{(\Delta x)^{2 / \alpha}}^{+\infty}\left[G\left(x_{2}, \tau\right)-G\left(x_{1}, \tau\right)\right] \\
& \times\left[\partial_{\tau}^{\alpha} g_{1}(t-\tau)-\partial_{\tau}^{\alpha} g_{1}(t)\right] d \tau \equiv \sum_{i=1}^{3} \Im_{i} .
\end{aligned}
$$

Using inequality (38) with $t:=(\Delta x)^{2 / \alpha}, \bar{\theta}:=\theta$, we obtain

$$
\left|\mathfrak{I}_{1}\right|+\left|\mathfrak{\Im}_{2}\right| \leq \text { const. }\left\langle\partial_{t}^{\alpha} g_{1}\right\rangle_{t,[0, T]}^{((\theta / 2) \alpha)}\left(x_{2}-x_{1}\right)^{\theta}
$$

As for the term $\mathfrak{\Im}_{3}$, we apply the mean-value theorem to the difference $\left[G\left(x_{2}, \tau\right)-G\left(x_{1}, \tau\right)\right]$ together with inequality (39) (where $\delta:=(\Delta x)^{2 / \alpha}, \bar{\theta}:=\theta$ ) and deduce

$$
\left|\Im_{3}\right| \leq \text { const. }\left\langle\partial_{t}^{\alpha} g_{1}\right\rangle_{t,[0, T]}^{((\theta / 2) \alpha)}(\Delta x)^{1+(2 / \alpha)(-(\alpha / 2)+\theta(\alpha / 2))} .
$$

Thus, representation (54) together with inequalities (55) and (56) prove the correctness of (44).

To complete the proof of Lemma 7, we need to obtain inequality (45). Let $t_{1}, t_{2} \in[0, T]$ and $t_{2}>t_{1}$. Denote

$$
\Delta t:=t_{2}-t_{1}, \quad \Delta_{t} \partial_{t}^{\alpha} v:=\partial_{t}^{\alpha} v\left(x, t_{2}\right)-\partial_{t}^{\alpha} v\left(x, t_{1}\right)
$$

We analyze the difference

$$
\begin{aligned}
\Delta_{t} \partial_{t}^{\alpha} v= & \int_{2 t_{1}-t_{2}}^{t_{1}} G\left(x, t_{1}-\tau\right)\left[\partial_{t}^{\alpha} g_{1}\left(t_{1}\right)-\partial_{t}^{\alpha} g_{1}(\tau)\right] d \tau \\
& +\int_{2 t_{1}-t_{2}}^{t_{2}} G\left(x, t_{2}-\tau\right)\left[\partial_{t}^{\alpha} g_{1}(\tau)-\partial_{t}^{\alpha} g_{1}\left(t_{2}\right)\right] d \tau \\
& +\int_{-\infty}^{2 t_{1}-t_{2}} G\left(x, t_{1}-\tau\right) d \tau\left[\partial_{t}^{\alpha} g_{1}\left(t_{1}\right)-\partial_{t}^{\alpha} g_{1}\left(t_{2}\right)\right] \\
& +\int_{-\infty}^{2 t_{1}-t_{2}}\left[\partial_{t}^{\alpha} g_{1}(\tau)-\partial_{t}^{\alpha} g_{1}\left(t_{2}\right)\right] \\
& \times\left[G\left(x, t_{2}-\tau\right)-G\left(x, t_{1}-\tau\right)\right] d \tau \\
& +\left[\partial_{t}^{\alpha} g_{1}\left(t_{2}\right)-\partial_{t}^{\alpha} g_{1}\left(t_{1}\right)\right] \equiv \sum_{i=1}^{5} \widetilde{\Im}_{i} .
\end{aligned}
$$

As for the last term in this sum, it is estimated by $(\Delta t)^{\theta \alpha / 2}\left\langle\partial_{t}^{\alpha} g_{1}\right\rangle_{t,[0, T]}^{(\theta / 2) \alpha}$. We change the variable $\tau=t_{1}-z$ in the term $\mathfrak{\Im}_{1}$ and apply estimate (38) with $t:=\Delta t, \bar{\theta}:=\theta$. Thus, we have

$$
\left|\mathfrak{J}_{1}\right| \leq \text { const. }(\Delta t)^{\theta \alpha / 2}\left\langle\partial_{t}^{\alpha} g_{1}\right\rangle_{t,[0, T]}^{((\theta / 2) \alpha)}
$$

In the same way, we evaluate the function $\mathfrak{\Im}_{2}$. The estimate of the term $\mathfrak{J}_{3}$ follows from the properties of the function $\partial_{t}^{\alpha} g_{1}$ and inequality (36). At last, the mean-value theorem together with estimate (40), where $\delta:=\Delta t, \bar{\theta}:=\theta$, lead to

$$
\left|\mathfrak{\Im}_{4}\right| \leq C(\Delta t)^{\theta \alpha / 2}\left\langle\partial_{t}^{\alpha} g_{1}\right\rangle_{t,[0, T]}^{((\theta / 2) \alpha)}
$$

Therefore, inequality (45) is deduced from (58)-(60).

Now, based on the results of Lemmas 6 and 7, we can infer the next assertion.

Lemma 8. Let conditions of Lemma 7 hold. Then there exists a unique solution $v(x, t) \in C^{2+\theta,((2+\theta) / 2) \alpha}\left(\bar{D}_{T}^{+}\right)$of problem $(22)-$ (24), which is represented with (32) and

$$
\|v\|_{C^{2+\theta,((2+\theta) / 2) \alpha}\left(\bar{D}_{T}^{+}\right)} \leq C\left\|g_{1}\right\|_{C^{((2+\theta) / 2) \alpha}([0, T])} \text {. }
$$

Proof. First of all we obtain estimate (61). One can get the following inequality using the results of Lemma 7 and (22), where $g_{0} \equiv 0$ :

$$
\begin{aligned}
\left\|\frac{\partial^{2} v}{\partial x^{2}}\right\|_{C^{\theta,(\theta / 2) \alpha}\left(\bar{D}_{T}^{+}\right)} \leq \text {const. }\left\|\partial_{t}^{\alpha} v\right\|_{C^{\theta,(\theta / 2) \alpha}\left(\bar{D}_{T}^{+}\right)} \\
\leq C\left\|g_{1}\right\|_{C^{((2+\theta) / 2) \alpha}([0, T])^{\prime}} .
\end{aligned}
$$

Next, we use formula (3.5.4) from [1] as follows:

$$
w(t)-w(0)=\frac{1}{\Gamma(\alpha)} \int_{0}^{t} \frac{\partial_{\tau}^{\alpha} w(\tau)}{(t-\tau)^{1-\alpha}} d \tau
$$

to evaluate the maximum of $|v(x, t)|$. Hence, (43) and (63) lead to inequality

$$
\sup _{\bar{D}_{T}^{+}}|v| \leq \text { const.sup } \bar{D}_{T}^{+}\left|\partial_{t}^{\alpha} v\right| \leq C\left\|g_{1}\right\|_{C^{((2+\theta) / 2) \alpha}([0, T])} ;
$$

here we use the fact that $v(x, 0)=0$.

After that, the minor seminorms of the function $v(x, t)$ are estimated with the interpolation inequalities from $\mathrm{Sec}-$ tion 8.8 [37] and (43)-(45), (62), and (64). Therefore, the arguments above prove inequality (61) and the embedding $v(x, t) \in C^{2+\theta,((2+\theta) / 2) \alpha}\left(\bar{D}_{T}^{+}\right)$.

Next, we show that the function $v(x, t)$ given by (32) satisfies (22). To this end, we use equality (46) and (48) and represent $\partial_{t}^{\alpha} v(x, t)$ as

$$
\begin{aligned}
& \partial_{t}^{\alpha} v(x, t) \\
& \quad=\int_{0}^{t} g_{1}(t-\tau) D_{t}^{\alpha} G(x, \tau) d \tau+g_{1}(t) \lim _{\tau \rightarrow 0} I_{\tau}^{1-\alpha} G(x, \tau) .
\end{aligned}
$$


Then (41) leads to

$$
\partial_{t}^{\alpha} v(x, t)=\int_{0}^{t} g_{1}(t-\tau) D_{t}^{\alpha} G(x, \tau) d \tau .
$$

Next, due to property (42) of the function $G(x, t)$, one can check that

$$
\partial_{t}^{\alpha} v(x, t)=a_{0} \frac{\partial^{2} v}{\partial x^{2}}
$$

As it follows from the first inequality in (37), the function $v(x, t)$ represented by (32) satisfies the following conditions:

$$
\lim _{t \rightarrow 0} v(x, t)=0 ; \quad v(x, t) \longrightarrow 0 \quad \text { if } x \longrightarrow+\infty .
$$

Finally, it is necessary to show that the function $v(x, t)$ meets boundary condition (24). To this end, we observe the next difference (here we will essentially use statement (35))

$$
\begin{aligned}
& \left|v(x, t)-g_{1}(t)\right| \\
& \leq\left|\int_{x}^{+\infty} G(x, \tau)\left[g_{1}(t-\tau)-g_{1}(t)\right] d \tau\right| \\
& \quad+\left|\int_{0}^{x} G(x, \tau)\left[g_{1}(t-\tau)-g_{1}(t)\right] d \tau\right| \\
& \leq \operatorname{sip}_{[0, T]}\left|g_{1}\right| \int_{x}^{+\infty} G(x, \tau) d \tau+\left\langle g_{1}\right\rangle_{t,[0, T]}^{((\theta / 2) \alpha)} \\
& \quad \times \int_{0}^{x} G(x, \tau) \tau^{\alpha \theta / 2} d \tau \equiv A_{1}+A_{2} .
\end{aligned}
$$

Applying inequality (38) with $t:=x, \bar{\theta}:=\theta$ to the term $A_{2}$, we get

$$
\left|A_{2}\right| \leq \text { const. }\left\|g_{1}\right\|_{C^{(2+\theta) / 2) \alpha([0, T])}} x^{\alpha \theta / 2} .
$$

To estimate the term $A_{1}$ in (69), we use the Wright formula (see [38] or (2.2.5) in [21]):

$$
-\beta z W(z ; \beta, 1-\beta)=W(z, \beta, 0),
$$

and rewrite the function $G(x, t)$ as

$$
G(x, t)=\frac{\alpha}{2 \sqrt{a_{0}}} x t^{-1-(\alpha / 2)} W\left(-\frac{x}{\sqrt{a_{0}}} t^{-\alpha / 2} ;-\frac{\alpha}{2} ; 1-\frac{\alpha}{2}\right) .
$$

After that, due to Lemmas 2.2.4 and 2.2.7 in [21] and representation (72), we have

$$
0<G(x, t)<\text { const. } x t^{-1-\alpha / 2} .
$$

Thus,

$$
\begin{aligned}
\left|A_{1}\right| & \leq \underset{[0, T]}{\text { const.sup }}\left|g_{1}\right| \int_{x}^{+\infty} x \tau^{-1-\alpha / 2} d \tau \\
& \leq \text { const.sup }\left|g_{1}\right| x^{1-\alpha / 2} .
\end{aligned}
$$

Then, we joint estimates (69)-(74) and obtain

$$
\begin{aligned}
\left|v(x, t)-g_{1}(t)\right| & \\
\leq \text { const. }\left\|g_{1}\right\|_{C^{((\theta / 2) \alpha) \alpha}([0, T])}\left(x^{1-\alpha / 2}+x^{\alpha \theta / 2}\right) & \longrightarrow 0 \\
\text { if } x & \longrightarrow 0,
\end{aligned}
$$

which means that $\lim _{x \rightarrow 0} v(x, t)=g_{1}(t)$.

Therefore, as it was written above, the function $v(x, t)$ given by (32) is a solution of (22)-(24) in the case of (27). The uniqueness of this solution is proved like the arguments of Theorem 3.2 from [39].

Now we remove restriction (27). To this end, it is enough to consider the Cauchy problem:

$$
\begin{gathered}
\partial_{t}^{\alpha} \bar{v}(x, t)-a_{0} \frac{\partial^{2} \bar{v}(x, t)}{\partial x^{2}}=\bar{g}_{0}(x, t) \\
\text { in } R^{1} \times(0, T), \alpha \in(0,1) ; \\
\bar{v}(x, 0)=\bar{v}_{0}(x), \quad x \in R^{1} ; \\
\bar{v}(x, t) \longrightarrow 0, \quad \text { if }|x| \longrightarrow+\infty, \forall t \geq 0 .
\end{gathered}
$$

Here, $\bar{g}_{0}(x, t)$ and $v_{0}(x)$ are extensions of the functions $g_{0}(x, t)$ and $v_{0}(x)$, correspondingly, onto $x<0$. These functions together with their corresponding derivatives have finite supports and

$$
\begin{gathered}
\left\|\bar{g}_{0}\right\|_{C^{\theta,(\theta / 2) \alpha}\left(R^{1} \times[0, T]\right)} \leq C\left\|g_{0}\right\|_{C^{\theta,(\theta / 2) \alpha}\left(\bar{D}_{T}^{+}\right)}, \\
\left\|\bar{v}_{0}\right\|_{C^{2+\theta}\left(R^{1}\right)} \leq C\left\|v_{0}\right\|_{C^{2+\theta}\left(\bar{D}^{+}\right)} .
\end{gathered}
$$

The results of Theorem 3.2 from [39] give the one-valued solvability of (76) and

$$
\begin{aligned}
& \|\bar{v}\|_{C^{2+\theta,((2+\theta) / 2) \alpha\left(R^{1} \times[0, T]\right)}} \\
& \quad \leq C\left(\left\|\bar{g}_{0}\right\|_{C^{\theta,(\theta / 2) \alpha}\left(\bar{D}_{T}^{+}\right)}+\left\|\bar{v}_{0}\right\|_{C^{2+\theta}\left(\bar{D}^{+}\right)}\right),
\end{aligned}
$$

or, due to inequalities (77),

$$
\begin{aligned}
& \|\bar{v}\|_{C^{2+\theta,((2+\theta) / 2) \alpha}\left(R^{1} \times[0, T]\right)} \\
& \quad \leq C\left(\left\|g_{0}\right\|_{C^{\theta,(\theta / 2) \alpha}\left(\bar{D}_{T}^{+}\right)}+\left\|v_{0}\right\|_{C^{2+\theta}\left(\bar{D}^{+}\right)}\right) .
\end{aligned}
$$

After that, we will look for the solution $v(x, t)$ of problem (22)-(24) as

$$
v(x, t)=\bar{v}(x, t)+\overline{\bar{v}}(x, t), \quad(x, t) \in \bar{D}_{T}^{+},
$$

where $\overline{\bar{v}}(x, t)$ satisfies conditions (22)-(24) with the new right-hand sides which meet requirements of Lemma 8 . Hence, we can apply the results of Lemma 8 to the function $\overline{\bar{v}}(x, t)$. This fact and the properties (see (77)) of the function $\bar{v}(x, t)$ allow us to obtain the next results. 
Theorem 9. Let $\alpha, \theta \in(0,1)$, and conditions (25) and (26) hold, and $g_{0} \in C^{\theta,(\theta / 2) \alpha}\left(\bar{D}_{T}^{+}\right), v_{0} \in C^{2+\theta}\left(\bar{D}^{+}\right), g_{1}(t) \in$ $C^{((2+\theta) / 2) \alpha}([0, T])$ for any positive number $T$. Then there exists a unique solution $v \in C^{2+\theta,((2+\theta) / 2) \alpha}\left(\bar{D}_{T}^{+}\right)$of problem $(22)-(24)$ and

$$
\begin{gathered}
\|v\|_{C^{2+\theta,((2+\theta) / 2) \alpha}\left(\bar{D}_{T}^{+}\right)} \\
\leq C\left(\left\|g_{1}\right\|_{C^{((2+\theta) / 2) \alpha}([0, T])}+\left\|g_{0}\right\|_{C^{\theta,(\theta / 2) \alpha}\left(\bar{D}_{T}^{+}\right)}\right. \\
\left.+\left\|v_{0}\right\|_{C^{2+\theta}\left(\bar{D}^{+}\right)}\right) .
\end{gathered}
$$

3.2. The Model Initial-Boundary Value Problem with a Fractional Dynamic Boundary Condition. Here we study the following problem: it is necessary to find the function $w(x, t)$ by the following conditions:

$$
\begin{gathered}
\partial_{t}^{\alpha} w(x, t)-a_{0} \frac{\partial^{2} w(x, t)}{\partial x^{2}}=\Phi_{0}(x, t) \quad \text { in } D_{T}^{+}, \alpha \in(0,1), \\
w(x, 0)=w_{0}(x), \quad x \in D^{+} ; \\
w(x, t) \longrightarrow 0 \quad \text { if } x \longrightarrow+\infty, \forall t \in[0, T], \\
\partial_{t}^{\alpha} w(0, t)-b_{0} \frac{\partial w(0, t)}{\partial x}=\Phi_{1}(t), \quad t \in[0, T]
\end{gathered}
$$

where $\Phi_{i}, i=0,1, w_{0}$ are the given functions. Let the following conditions hold:

$$
\begin{aligned}
& b_{0} \frac{\partial w_{0}(0)}{\partial x}+\Phi_{1}(0)=a_{0} \frac{\partial^{2} w_{0}(0)}{\partial^{2} x}+\Phi_{0}(0,0) \\
& w_{0}(x), \Phi_{0}(x, t) \equiv 0, \quad \text { if }|x|<R_{0} \forall t \in[0, T]
\end{aligned}
$$

for some positive number $R_{0}$.

At the beginning, we assume that

$$
w_{0}(x), \Phi_{0}(x, t) \equiv 0
$$

and search a solution of (82) under this restriction in the class $C^{2+\theta,((2+\theta) / 2) \alpha}\left(\bar{D}_{T}^{+}\right)$, if $\Phi_{1}(t) \in C^{((1+\theta) / 2) \alpha}([0, T]), \theta \in(0,1)$.

Note that conditions (83) and (84) allow us to extend the right-hand sides in (82) by 0 for $t \leq 0$. We save, for simplicity, the same notation for the extended function $\Phi_{1}(t)$.

After the application of the Laplace transformation in time $t$ to problem (82), we have the following:

$$
\begin{gathered}
p^{\alpha} \widehat{w}(x, p)-a_{0} \frac{\partial^{2} \widehat{w}(x, p)}{\partial x^{2}}=0, \quad x \in D^{+} ; \\
\widehat{w}(x, p) \longrightarrow 0 \quad \text { if } x \longrightarrow+\infty ; \\
p^{\alpha} \widehat{w}(0, p)-b_{0} \frac{\partial \widehat{w}(0, p)}{\partial x}=\widehat{\Phi}_{1}(p) .
\end{gathered}
$$

Here, we used again formula (30). Some simple calculations lead to the function

$$
\begin{aligned}
\widehat{w}(x, p) & =\frac{\widehat{\Phi}_{1}(p)}{p^{\alpha / 2}\left(p^{\alpha / 2}+b_{0} a_{0}^{-1 / 2}\right)} \exp \left(-x a_{0}^{-1 / 2} p^{\alpha / 2}\right) \\
& \equiv \widehat{F}(p) \exp \left(-x a_{0}^{-1 / 2} p^{\alpha / 2}\right),
\end{aligned}
$$

which is the solution of problem (85). Due to formulas (2.30) in [34] and (1.80) in [29], we obtain, after applying of the inverse Laplace transformation to (86), that

$$
w(x, t)=\int_{0}^{t} G(x, t-\tau) F(\tau) d \tau,
$$

where the kernel $G(x, t)$ is given by $(33)$ and

$$
\begin{gathered}
F(t)=\int_{0}^{t} G_{2}(\tau) \Phi_{1}(t-\tau) d \tau, \\
G_{2}(t)=t^{\alpha-1} E_{\alpha / 2, \alpha}\left(-b_{0} a_{0}^{-1 / 2} t^{\alpha / 2}\right) .
\end{gathered}
$$

Here, $E_{\gamma, \beta}(z)$ is the function of the Mittag-Leffler type, which is defined by the series expansion (see, e.g., (1.56) in [29] or (1.8.17) in [1])

$$
E_{\gamma, \beta}(z)=\sum_{k=0}^{\infty} \frac{z^{k}}{\Gamma(\gamma k+\beta)}, \quad \operatorname{Re} \gamma>0, \beta, z \in \mathbb{C} .
$$

Note that this two-parameter function of the Mittag-Leffler type was in fact introduced by Agarwal [40].

The function $G(x, t)$ has been studied in Section 3.1 (see Lemma 6). Thus, to describe the properties of the function $w(x, t)$, we have to observe the function $F(t)$. To this end, we will use the following properties of the kernel $G_{2}(t)$, which are proved in Appendix B.

Lemma 10. Let $N, \delta$, and $\theta$ be some positive constants, $\theta \in$ $(0,1) ; I_{t}^{v}$ and $D_{t}^{v}$ be the fractional Riemann-Liouville integral and derivative, correspondingly (their definitions are given in (49) and (47)). Then the following is true.

(i) $0 \leq G_{2}(t) \leq C \frac{t^{\alpha-1}}{1+t^{\alpha / 2}}, \quad \int_{0}^{t} G_{2}(\tau) d \tau \leq C t^{\alpha}$.

(ii) $\lim _{t \rightarrow 0} I_{t}^{1-\alpha} G_{2}(t)=1$.

(iii) $\int_{0}^{+\infty} D_{\tau}^{\alpha} G_{2}(\tau) d \tau=-1$

(iv) $\int_{0}^{t}\left|D_{\tau}^{\alpha} G_{2}(\tau)\right| \tau^{(1+\theta) \alpha / 2} d \tau \leq C t^{(1+\theta) \alpha / 2}$.

(v) $\int_{N}^{+\infty}\left|D_{\tau}^{\alpha} G_{2}(\tau)\right| d \tau \leq C$

(vi) $\left|\int_{\delta}^{+\infty} D_{\tau}^{\alpha} G_{2}(\tau) d \tau\right| \leq 1+\frac{C}{1+\delta^{\alpha / 2}}$.

(vii) $\int_{\delta}^{+\infty}\left|\frac{d}{d \tau} D_{\tau}^{\alpha} G_{2}(\tau)\right| \tau^{(1+\theta) \alpha / 2} d \tau \leq C \delta^{-1+\alpha(\theta+1) / 2}$. 
Lemma 11. Let $\Phi_{1}(t)$ meet requirement (83) and let $\Phi_{1} \epsilon$ $C^{((1+\theta) / 2) \alpha}([0, T])$. Then the function $F(t)$ represented by $(88)$ belongs to $C^{((3+\theta) / 2) \alpha}([0, T]), F(0)=0$, and the following estimate holds:

$$
\|F\|_{C^{((3+\theta) / 2) \alpha}([0, T])} \leq C\left\|\Phi_{1}\right\|_{C^{((1+\theta) / 2) \alpha}([0, T])} .
$$

Proof. First of all, we evaluate the value of $\max _{[0, T]}|F(t)|$. To this end, we use the first inequality in (90). Thus, one has

$$
\max _{[0, T]}|F(t)| \leq \max _{[0, T]}\left|\Phi_{1}(t)\right| \int_{0}^{t}\left|G_{2}(\tau)\right| d \tau \leq C \max _{[0, T]}\left|\Phi_{1}(t)\right| t^{\alpha} .
$$

This inequality gives that

$$
F(0)=0
$$

Next, we obtain the representation of $\partial_{t}^{\alpha} F(t)$. Due to equality (99) and properties (46) and (48), we conclude that

$$
\begin{aligned}
\partial_{t}^{\alpha} F(t) & =D_{t}^{\alpha} F(t) \\
& =\Phi_{1}(t) \lim _{t \rightarrow 0} I_{t}^{1-\alpha} G_{2}(t)+\int_{0}^{t} \Phi_{1}(t-\tau) D_{\tau}^{\alpha} G_{2}(\tau) d \tau
\end{aligned}
$$

Since $\Phi_{1}(0)=0$, we can rewrite the last equality as

$$
\begin{aligned}
& \partial_{t}^{\alpha} F(t) \\
& \quad=\Phi_{1}(t) \lim _{t \rightarrow 0} I_{t}^{1-\alpha} G_{2}(t)+\int_{0}^{+\infty} \Phi_{1}(t-\tau) D_{\tau}^{\alpha} G_{2}(\tau) d \tau,
\end{aligned}
$$

or, applying (91) and (92), we have

$$
\partial_{t}^{\alpha} F(t)=\int_{0}^{+\infty}\left[\Phi_{1}(t-\tau)-\Phi_{1}(t)\right] D_{\tau}^{\alpha} G_{2}(\tau) d \tau .
$$

To estimate $\max _{[0, T]}\left|\partial_{t}^{\alpha} F(t)\right|$, we use representation (102) and get

$$
\begin{aligned}
\left|\partial_{t}^{\alpha} F(t)\right| \leq & \int_{0}^{N} \tau^{(1+\theta) \alpha / 2}\left|D_{\tau}^{\alpha} G_{2}(\tau)\right| d \tau\left\langle\Phi_{1}\right\rangle_{t,[0, T]}^{((1+\theta / 2) \alpha)} \\
& +2 \max _{[0, T]}\left|\Phi_{1}(t)\right| \int_{N}^{+\infty}\left|D_{\tau}^{\alpha} G_{2}(\tau)\right| d \tau
\end{aligned}
$$

After that, we apply inequality (93) with $t:=\delta$ to the first term in (103) and (94) to the second and get

$$
\max _{[0, T]}\left|\partial_{t}^{\alpha} F(t)\right| \leq C\left\|\Phi_{1}\right\|_{C^{((1+\theta) / 2) \alpha}([0, T])} .
$$

Finally, to complete the proof of Lemma 11 is necessary to estimate of $\left\langle\partial_{t}^{\alpha} F\right\rangle_{t,[0, T]}^{(((1+\theta) / 2) \alpha)}$. Let $t_{2}, t_{1} \in[0, T]$ and $t_{2}>t_{1}$. Denote

$$
\Delta t:=t_{2}-t_{1}, \quad \Delta_{t} \partial_{t}^{\alpha} F=\partial_{t}^{\alpha} F\left(t_{2}\right)-\partial_{t}^{\alpha} F\left(t_{1}\right) .
$$

Using formula (102), we represent the difference $\Delta_{t} \partial_{t}^{\alpha} F$ as

$$
\begin{aligned}
\Delta_{t} \partial_{t}^{\alpha} F= & \int_{t_{1}-\Delta t}^{t_{2}} D_{\tau}^{\alpha} G_{2}\left(t_{2}-\tau\right)\left[\Phi_{1}(\tau)-\Phi_{1}\left(t_{2}\right)\right] d \tau \\
& +\int_{t_{1}-\Delta t}^{t_{1}} D_{\tau}^{\alpha} G_{2}\left(t_{1}-\tau\right)\left[\Phi_{1}\left(t_{1}\right)-\Phi_{1}(\tau)\right] d \tau \\
& +\int_{-\infty}^{t_{1}-\Delta t}\left[D_{\tau}^{\alpha} G_{2}\left(t_{2}-\tau\right)-D_{\tau}^{\alpha} G_{2}\left(t_{1}-\tau\right)\right] \\
& +\int_{-\infty}^{t_{1}-\Delta t} D_{\tau}^{\alpha} G_{2}\left(t_{1}-\tau\right) d \tau\left[\Phi_{1}\left(\Phi_{1}\right)-\Phi_{1}\left(t_{2}\right)\right] d \tau \\
\equiv & \sum_{i=1}^{4} \mathfrak{B}_{i} .
\end{aligned}
$$

Changing the variable $t_{2}-\tau=z$ in $\mathfrak{B}_{1}$ and $t_{1}-\tau=z$ in $\mathfrak{B}_{2}$, we get

$$
\mathfrak{B}_{i}=\int_{0}^{2 \Delta t} D_{\tau}^{\alpha} G_{2}(z)\left[\Phi_{1}\left(t_{i}-z\right)-\Phi_{1}\left(t_{i}\right)\right] d z, \quad i=1,2 .
$$

Then, the property of the function $\Phi_{1}(t)$ together with inequality (93) (where $t:=2 \Delta t$ ) lead to

$$
\left|\mathfrak{B}_{1}\right|+\left|\mathfrak{B}_{2}\right| \leq C\left\|\Phi_{1}\right\|_{C^{((1+\theta) / 2) \alpha}([0, T])}(\Delta t)^{(1+\theta) \alpha / 2} .
$$

To get the same estimate for the term $\mathfrak{B}_{3}$, we apply the meanvalue theorem to the difference $\left[D_{\tau}^{\alpha} G_{2}\left(t_{2}-\tau\right)-D_{\tau}^{\alpha} G_{2}\left(t_{1}-\tau\right)\right]$ and inequality (96) and have after some simple calculations

$$
\left|\mathfrak{B}_{3}\right| \leq C\left\|\Phi_{1}\right\|_{C^{(1+\theta) / 2) \alpha}([0, T])}(\Delta t)^{(1+\theta) \alpha / 2} .
$$

As for the estimate of $\mathfrak{B}_{4}$, one follows immediately from (95) where $\delta:=\Delta t$ and properties of the function $\Phi_{1}$ are

$$
\begin{aligned}
\left|\mathfrak{B}_{4}\right| & \leq \text { const. }(\Delta t)^{(1+\theta) \alpha / 2}\left|\int_{\Delta t}^{+\infty} D_{\tau}^{\alpha} G_{2}(\tau) d \tau\right| \\
& \leq C\left\|\Phi_{1}\right\|_{C^{((1+\theta) / 2) \alpha}([0, T])}(\Delta t)^{(1+\theta) \alpha / 2} .
\end{aligned}
$$

Hence, inequalities (106)-(110) lead to estimate

$$
\left\langle\partial_{t}^{\alpha} F\right\rangle_{t,[0, T]}^{(((1+\theta) / 2) \alpha)} \leq C\left\|\Phi_{1}\right\|_{C^{((1+\theta) / 2) \alpha}([0, T])}(\Delta t)^{(1+\theta) \alpha / 2},
$$

which completes the proof of Lemma 11.

Due to results of this lemma and arguments like (70)(74), we can infer that

$$
\begin{gathered}
\left.w(x, t)\right|_{\partial D_{T}^{+}}=F(t),\left.\quad \partial_{t}^{\alpha} w(x, t)\right|_{\partial D_{T}^{+}}=\partial_{t}^{\alpha} F(t), \\
w(x, t) \in C^{((3+\theta) / 2) \alpha}\left(\partial D_{T}^{+}\right) .
\end{gathered}
$$

Based on the results of Lemma 11, properties of the function $G(x, t)$ (see Lemmas 7 and 8 ), and (112), we can get the next assertion. 
Lemma 12. Let conditions (83) and (84) hold and let $\Phi_{1}(t)$ meet the requirements of Lemma 11. Then the function $w(x, t)$ represented by (87) satisfies (112) and

$$
\begin{gathered}
\|w\|_{C^{((3+\theta) / 2) \alpha}\left(\partial D_{T}^{+}\right)}+\left\|\partial_{t}^{\alpha} w\right\|_{C^{\theta,(\theta / 2) \alpha}\left(\bar{D}_{T}^{+}\right)} \\
\leq C\left\|\Phi_{1}\right\|_{C^{((1+\theta) / 2) \alpha}([0, T])} .
\end{gathered}
$$

Now, we can prove the solvability of problem (82).

Theorem 13. Let $\theta, \alpha \in(0,1)$, and conditions (83) hold, and $\Phi_{0}(x, t) \in C^{\theta,(\theta / 2) \alpha}\left(\bar{D}_{T}^{+}\right), w_{0}(x) \in C^{2+\theta}\left(\bar{D}^{+}\right), \Phi_{1}(t) \in$ $C^{((1+\theta) / 2) \alpha}([0, T])$ for any positive number $T$. Then there exists a unique solution $w(x, t) \in C^{2+\theta,((2+\theta) / 2) \alpha}\left(\bar{D}_{T}^{+}\right)$of problem $(82)$ and

$$
\begin{gathered}
\|w\|_{C^{2+\theta,((2+\theta) / 2) \alpha}\left(\bar{D}_{T}^{+}\right)}+\left\|\partial_{t}^{\alpha} w\right\|_{C^{((1+\theta) / 2) \alpha}\left(\partial D_{T}^{+}\right)} \\
\leq C\left[\left\|\Phi_{0}\right\|_{C^{\theta,(\theta / 2) \alpha}\left(\bar{D}_{T}^{+}\right)}+\left\|\Phi_{1}\right\|_{C^{((1+\theta) / 2) \alpha}([0, T])}\right. \\
\left.+\left\|w_{0}\right\|_{C^{2+\theta}\left(\bar{D}^{+}\right)}\right] .
\end{gathered}
$$

Proof. To prove this theorem in the case of (84) it is enough to consider the following Dirichlet problem:

$$
\begin{gathered}
\partial_{t}^{\alpha} w(x, t)-a_{0} \frac{\partial^{2} w(x, t)}{\partial x^{2}}=0 \quad \text { in } D_{T}^{+}, \alpha \in(0,1) ; \\
w(x, 0)=0, \quad x \in D^{+} ; \\
w(x, t) \longrightarrow 0 \quad \text { if } x \longrightarrow+\infty, \quad \forall t \in[0, T] ; \\
w(0, t)=F(t), \quad t \in[0, T]
\end{gathered}
$$

and to apply the results of Theorem 9 and Lemma 12.

To remove restriction (84), we will look for the solution of problem (82) in the following form:

$$
w(x, t)=w_{1}(x, t)+w_{2}(x, t)
$$

where $w_{1}(x, t)$ is the solution of the following Dirichlet problem:

$$
\begin{gathered}
\partial_{t}^{\alpha} w_{1}(x, t)-a_{0} \frac{\partial^{2} w_{1}(x, t)}{\partial x^{2}} \\
=\Phi_{0}(x, t) \text { in } D_{T}^{+}, \alpha \in(0,1) ; \\
w_{1}(x, 0)=w_{0}(x), \quad x \in D^{+} ; \\
w_{1}(x, t) \longrightarrow 0 \quad \text { if } x \rightarrow+\infty, \forall t \in[0, T] ; \\
w_{1}(0, t)=0, \quad t \in[0, T]
\end{gathered}
$$

and the function $w_{2}(x, t)$ solves the following problem:

$$
\begin{gathered}
\partial_{t}^{\alpha} w_{2}(x, t)-a_{0} \frac{\partial^{2} w_{2}(x, t)}{\partial x^{2}}=0 \quad \text { in } D_{T}^{+}, \quad \alpha \in(0,1) ; \\
w_{2}(x, t) \underset{x \rightarrow+\infty}{\longrightarrow} 0 \quad \forall t \in[0, T] \\
w_{2}(x, 0)=0, \quad x \in D^{+} ; \\
\partial_{t}^{\alpha} w_{2}(0, t)-b_{0} \frac{\partial w_{2}(0, t)}{\partial x}=\Phi_{1}(t)+b_{0} \frac{\partial w_{1}(0, t)}{\partial x} \equiv \bar{\Phi}_{1}(t), \\
t \in[0, T] .
\end{gathered}
$$

The one-valued solvability of problem (117) follows from Theorem 9, which gives

$$
\begin{array}{r}
\left\|w_{1}\right\|_{C^{2+\theta,((2+\theta) / 2) \alpha}\left(\bar{D}_{T}^{+}\right)}+\left\|\partial_{t}^{\alpha} w_{1}\right\|_{C^{((1+\theta) / 2) \alpha}\left(\partial D_{T}^{+}\right)} \\
\leq C\left[\left\|\Phi_{0}\right\|_{C^{\theta,(\theta / 2) \alpha}\left(\bar{D}_{T}^{+}\right)}+\left\|w_{0}\right\|_{C^{2+\theta}\left(\bar{D}^{+}\right)}\right], \\
\partial_{t}^{\alpha} w_{1}(0, t)=0, \quad t \in[0, T] .
\end{array}
$$

These properties of the function $w_{1}(x, t)$ allow us to conclude that $\bar{\Phi}_{1}(t) \in C^{((1+\theta) / 2)}([0, T])$ and $\bar{\Phi}_{1}(0)=0$; that is, $\bar{\Phi}_{1}(t)$ satisfies the conditions of Lemma 12, and the right-hand sides of (118) meet requirement (84). It means that there exists a unique solution $w_{2} \in C^{2+\theta,((2+\theta) / 2) \alpha}\left(\bar{D}_{T}^{+}\right)$of (118) which satisfies to inequality (119) with $\Phi_{0}(x, t)=w_{0}(x) \equiv 0$.

Finally, returning to representation (116) for $w(x, t)$ and using the described properties of $w_{i}(t, x), i=1,2$, we complete the proof of Theorem 13.

\section{The Proofs of Theorems 3-5}

Note that the proofs of Theorems 4 and 5 are analogous to the one of Theorem 3 and use the technique from Chapter 4 [32] together with the results of the solvability to the model problems from Section 3. That is why, we represent here only the proof of Theorem 3 . In this route, we will need the solvability in $C^{2+\theta,((2+\theta) / 2) \alpha}\left(\bar{\Omega}_{T}\right)$ of the next initial-boundary value problem:

$$
\begin{gathered}
\partial_{t}^{\alpha} U(x, t)-a_{0}(x, t) \frac{\partial^{2} U(x, t)}{\partial x^{2}}+a_{1}(x, t) \frac{\partial U(x, t)}{\partial x} \\
+a_{2}(x, t) U(x, t)=g_{0}(x, t), \\
\quad(x, t) \in \Omega_{T}, \alpha \in(0,1] ; \\
U(x, t)=g_{i}(t) \quad \text { on } \Gamma_{i T}, i=1,2 ; \\
U(x, 0)=U_{0}(x) \quad \text { in } \Omega .
\end{gathered}
$$

Reaching this goal is enough to repeat the arguments of Section 3.3 from [39] and apply the results of Theorem 9. Thus, we can assert the following. 
Theorem 14. Let $\theta, \alpha \in(0,1)$ and the following conditions

$$
\begin{gathered}
g_{1}(0)=U_{0}(a) ; \\
\partial_{t}^{\alpha} g_{1}(0)=a_{0}(a, 0) \frac{\partial^{2} U_{0}(a)}{\partial x^{2}}-a_{1}(a, 0) \frac{\partial U_{0}(a)}{\partial x} \\
-a_{2}(a, 0) U_{0}(a)+g_{0}(a, 0) ; \\
g_{2}(0)=U_{0}(b) ; \\
\partial_{t}^{\alpha} g_{2}(0)=a_{0}(b, 0) \frac{\partial^{2} U_{0}(b)}{\partial x^{2}}-a_{1}(b, 0) \frac{\partial U_{0}(b)}{\partial x} \\
-a_{2}(b, 0) U_{0}(b)+g_{0}(b, 0)
\end{gathered}
$$

hold. Let and $g_{0}(x, t) \in C^{\theta,(\theta / 2) \alpha}\left(\bar{\Omega}_{T}\right), U_{0}(x) \in C^{2+\theta}(\bar{\Omega})$, $g_{i}(t) \in C^{((2+\theta) / 2) \alpha}([0, T]), i=1,2$, for any positive number $T$. Then there exists a unique solution $u(x, t)$ of problem (120): $U(x, t) \in C^{2+\theta,((2+\theta) / 2) \alpha}\left(\bar{\Omega}_{T}\right)$, and

$$
\begin{aligned}
\|U\|_{C^{2+\theta,((2+\theta) / 2) \alpha}\left(\bar{\Omega}_{T}\right)} & \\
\leq C & {\left[\left\|g_{0}\right\|_{C^{\theta,(\theta / 2) \alpha}\left(\bar{\Omega}_{T}\right)}+\left\|U_{0}\right\|_{C^{2+\theta}(\bar{\Omega})}\right.} \\
& \left.+\sum_{i=1}^{2}\left\|g_{i}\right\|_{C^{((2+\theta) / 2) \alpha}([0, T])}\right]
\end{aligned}
$$

where a positive constant $C$ depends only on the measure of $\Omega$ and $\left\|a_{i}\right\|_{C^{\theta,(\theta / 2) \alpha}\left(\bar{\Omega}_{T}\right)}, i=\overline{0,2}$.

At the beginning, we prove Theorem 9 in the case of $t \in[0, \tau]$ for some small $\tau<\tau_{0}$. Then, we will show how the solution $u(x, t)$ is extended from $[0, \tau]$ into $[0, T]$, where $T=\tau+\tau_{0}$, for all $\tau_{0}>0$.

Lemma 15. Let the conditions of Theorem 3 hold. Then there exists a unique solution $u(x, t) \in C^{2+\theta,((2+\theta) / 2) \alpha}\left(\bar{\Omega}_{\tau}\right)$ of problem (1)-(5), for any $\tau \in\left(0, \tau_{0}\right]$, and

$$
\begin{gathered}
\|u\|_{C^{2+\theta,((2+\theta) / 2) \alpha}\left(\bar{\Omega}_{\tau}\right)}+\left\|\partial_{t}^{\alpha} u\right\|_{C^{((1+\theta) / 2) \alpha}\left(\Gamma_{2 \tau}\right)} \\
\leq C\left[\left\|f_{0}\right\|_{C^{\theta,(\theta / 2) \alpha}\left(\bar{\Omega}_{\tau}\right)}+\left\|u_{0}\right\|_{C^{2+\theta}(\bar{\Omega})}\right. \\
\left.+\left\|f_{1}\right\|_{C^{((1+\theta) / 2) \alpha}\left(\Gamma_{2 \tau}\right)}+\left\|f_{2}\right\|_{C^{((2+\theta) / 2) \alpha}\left(\Gamma_{1 \tau}\right)}\right],
\end{gathered}
$$

where the positive constant $C$ depends on $\left\|a_{i}\right\|_{C^{\theta,(\theta / 2) \alpha}\left(\bar{\Omega}_{\tau}\right)}, i=$ $\overline{0,2},\left\|b_{j}\right\|_{C^{(1+\theta) / 2) \alpha}\left(\Gamma_{2 \tau}\right)}, j=0,1$, and the measure of $\Omega$.

Proof. If the right-hand sides of (1)-(5) meet the following requirements:

$$
\begin{gathered}
f_{0}(x, t) \in C_{0}^{\theta,(\theta / 2) \alpha}\left(\bar{\Omega}_{\tau}\right), \quad f_{1}(t) \in C_{0}^{((1+\theta) / 2) \alpha}\left(\Gamma_{2 \tau}\right), \\
f_{2}(t) \in C_{0}^{((2+\theta) / 2) \alpha}\left(\Gamma_{1 \tau}\right), \quad u_{0}(x) \equiv 0 ;
\end{gathered}
$$

then by repeating the arguments of $\$ 4-\$ 7$ from Chapter 4 [32] and using the results of Theorems 9 and 13 and Theorem 3.2 from [39], we have proved the assertion of Lemma 15.
To remove restriction (124), we look for the solution of problem (1)-(5) as

$$
u(x, t)=U(x, t)+V(x, t)
$$

where the function $U(x, t)$ is the solution of problem (120) with

$$
\begin{gathered}
g_{0}(x, t):=f_{0}(x, t) ; \quad U_{0}(x):=u_{0}(x) ; \\
g_{1}(t):=u_{0}(a)+U_{1}(a) \frac{t^{\alpha}}{\alpha \Gamma(\alpha)} ; \\
g_{2}(t):=u_{0}(b)+U_{1}(b) \frac{t^{\alpha}}{\alpha \Gamma(\alpha)} ; \\
U_{1}(x)=f_{0}(x, 0)+a_{0}(x, 0) \frac{\partial^{2} u_{0}(x)}{\partial x^{2}} \\
\quad-a_{1}(x, 0) \frac{\partial u_{0}(x)}{\partial x}-a_{2}(x, 0) u_{0}(x)
\end{gathered}
$$

and the unknown function $V(x, t)$ is the solution of the problem

$$
\begin{aligned}
& \partial_{t}^{\alpha} V(x, t)-a_{0}(x, t) \frac{\partial^{2} V(x, t)}{\partial x^{2}}+a_{1}(x, t) \frac{\partial V(x, t)}{\partial x} \\
& +a_{2}(x, t) V(x, t)=0 \quad \text { in } \Omega_{\tau} \text {; } \\
& V(x, 0)=0 \quad \text { in } \Omega \text {; } \\
& V(x, t)=f_{2}(t)-g_{1}(t) \\
& \equiv f_{2}^{*}(t) \text { on } \Gamma_{1 \tau} \\
& \partial_{t}^{\alpha} V(x, t)+b_{0}(t) \frac{\partial V(x, t)}{\partial x}+b_{1}(t) V(x, t) \\
& =f_{1}(t)-\partial_{t}^{\alpha} g_{2}(t)-b_{0}(t) \frac{\partial U(b, t)}{\partial x}-b_{1}(t) g_{2}(t) \\
& \equiv f_{1}^{*}(t) \quad \text { on } \Gamma_{2 \tau} \text {. }
\end{aligned}
$$

Our further arguments are divided into two parts. In the first step, we will show that the right-hand sides of (126) meet the requirements of Theorem 14, which will ensure the existence of the unique solution $U(x, t) \in C^{2+\theta,((2+\theta) / 2) \alpha}\left(\bar{\Omega}_{\tau}\right)$. The next step deals with the proving of the following equalities:

$$
f_{i}^{*}(0)=0, \quad i=1,2 .
$$

We obtain after simple calculations the following properties for the functions $g_{i}(t)$, for all $t \in[0, \tau]$ :

$$
\begin{array}{cc}
g_{1}(0)=u_{0}(a), \quad & g_{2}(0)=u_{0}(b) ; \\
\partial_{t}^{\alpha} g_{1}(t)=U_{1}(a), \quad \partial_{t}^{\alpha} g_{2}(t)=U_{2}(b) ; \\
g_{i}(t) \in C^{((2+\theta) / 2) \alpha}([0, \tau]), \quad i=1,2 .
\end{array}
$$

After that, using (129), one can easily check that the right-hand sides in (126) satisfy conditions of Theorem 14. 
That is why, there exists a unique solution $U(x, t) \quad \epsilon$ $C^{2+\theta,((2+\theta) / 2) \alpha}\left(\bar{\Omega}_{\tau}\right)$ as follows as follows:

$$
\begin{gathered}
\|U\|_{C^{2+\theta,((2+\theta) / 2) \alpha}\left(\bar{\Omega}_{\tau}\right)} \leq C\left[\left\|f_{0}\right\|_{C^{\theta,(\theta / 2) \alpha}\left(\bar{\Omega}_{\tau}\right)}+\left\|u_{0}\right\|_{C^{2+\theta}(\bar{\Omega})}\right], \\
\frac{\partial U(b, 0)}{\partial x}=\frac{\partial u_{0}(b)}{\partial x},
\end{gathered}
$$

where the positive constant $C$ depends on $\left\|a_{i}\right\|_{C^{\theta,(\theta / 2) \alpha}\left(\bar{\Omega}_{\tau}\right)}, i=$ $\overline{0,2}$, and the measure of $\Omega$.

Then we return to problem (127). Using properties (129) and (131), we get that

$$
\begin{gathered}
f_{2}^{*}(0)=f_{2}(0)-u_{0}(a) \\
f_{1}^{*}(0)=f_{1}(0)-b_{0}(0) \frac{\partial u_{0}(b)}{\partial x}-b_{1}(0) u_{0}(b)-f_{0}(b, 0) \\
+a_{2}(b, 0) u_{0}(b)-a_{0}(b, 0) \frac{\partial^{2} u_{0}(b)}{\partial x^{2}} \\
+a_{1}(b, 0) \frac{\partial u_{0}(b)}{\partial x} .
\end{gathered}
$$

After that, to get statement (128) is enough to apply condition (15) to the right-hand sides of equalities (132).

Finally, taking into account (129)-(131), we obtain the following statements:

$$
\begin{gathered}
\left\|f_{1}^{*}\right\|_{C^{((1+\theta) / 2) \alpha}([0, \tau])}+\left\|f_{2}^{*}\right\|_{C^{((2+\theta) / 2) \alpha}([0, \tau])} \\
\leq C\left[\left\|f_{0}\right\|_{C^{\theta,(\theta / 2) \alpha}\left(\bar{\Omega}_{\tau}\right)}+\left\|u_{0}\right\|_{C^{2+\theta}(\bar{\Omega})}\right. \\
\left.+\left\|f_{1}\right\|_{C^{((1+\theta) / 2) \alpha}([0, \tau])}+\left\|f_{2}\right\|_{C^{((2+\theta) / 2) \alpha}([0, \tau])}\right],
\end{gathered}
$$

where the positive constant $C$ depends on $\left\|a_{i}\right\|_{C^{\theta,(\theta / 2) \alpha}\left(\bar{\Omega}_{\tau}\right)}, i=$ $\overline{0,2},\left\|b_{j}\right\|_{C^{((1+\theta) / 2) \alpha}([0, \tau])}, j=0,1$, and the measure of $\Omega$.

Therefore, the right-hand sides of (127) meet requirements (124). It means that there exists a unique solution $V(x, t)$ of problem $(127)$ and that $V(x, t)$ satisfies to inequality (123) with the corresponding right-hand sides. This fact together with the properties of the function $U(x, t)$ (see (130)) and representation (125) completes the proof of Lemma 15.

Now we will extend the obtained solution $u(x, t), t \in$ $[0, \tau]$, in Lemma 15 , into $[\tau, T]$. In other words, we construct the function $\bar{U}(x, t) \in C^{2+\theta,((2+\theta) / 2) \alpha}\left(\bar{\Omega}_{T}\right), \partial_{t}^{\alpha} \bar{U}(x, t) \quad \epsilon$ $C^{((1+\theta) / 2) \alpha}\left(\Gamma_{2 T}\right)$ as follows:

$$
\begin{aligned}
& \partial_{t}^{\alpha} \bar{U}(x, t)-a_{0}(x, t) \frac{\partial^{2} \bar{U}(x, t)}{\partial x^{2}}+a_{1}(x, t) \frac{\partial \bar{U}(x, t)}{\partial x} \\
& \quad+a_{2}(x, t) \bar{U}(x, t)=f_{0}(x, t) \quad \text { in } \Omega_{T} ; \\
& \bar{U}(x, t)=f_{2}(t) \quad \text { on } \Gamma_{1 T} ; \\
& \partial_{t}^{\alpha} \bar{U}(x, t)+b_{0}(t) \frac{\partial \bar{U}(x, t)}{\partial x}+b_{1}(t) \bar{U}(x, t)=f_{1}(t) \quad \text { on } \Gamma_{2 T} ; \\
& \bar{U}(x, t)=u(x, t), \quad x \in \bar{\Omega}, t \in[0, \tau] ; \\
& \partial_{t}^{\alpha} \bar{U}(b, t)=\partial_{t}^{\alpha} u(b, t), \quad t \in[0, \tau],
\end{aligned}
$$

where the function $u(x, t)$ is constructed in Lemma 15; that is, the function $u(x, t): u(x, t) \in C^{2+\theta,((2+\theta) / 2) \alpha}\left(\bar{\Omega}_{\tau}\right), \partial_{t}^{\alpha} u(x, t) \in$ $C^{((1+\theta) / 2) \alpha}\left(\Gamma_{2 \tau}\right)$, is the unique solution of (1)-(5) for $t \in[0, \tau]$, $\tau<\tau_{0}$.

First, we assume that $T=2 \tau$ and consider problem (120) with the following right-hand sides:

$$
\begin{gathered}
g_{0}(x, t):=f_{0}(x, t) ; \quad U_{0}(x):=u_{0}(x) ; \\
g_{1}(t):=f_{2}(t) ; \\
g_{2}(t):=u_{0}(b)+\frac{1}{\Gamma(\alpha)} \int_{0}^{t} \tau^{\alpha-1} g(t-\tau) d \tau,
\end{gathered}
$$

where

$$
g(t)= \begin{cases}\partial_{t}^{\alpha} u(b, t), & \text { if } t \in[0, \tau] \\ \partial_{t}^{\alpha} u(b, \tau), & \text { if } t>\tau .\end{cases}
$$

After some calculations, we can infer that

$$
\begin{gathered}
\partial_{t}^{\alpha} g_{2}(t)=g(t) ; \quad g_{2}(0)=u_{0}(b) ; \\
\partial_{t}^{\alpha} g_{2}(0)=f_{0}(b, 0)+a_{0}(b, 0) \frac{\partial^{2} u_{0}(b)}{\partial x^{2}} \\
\quad-a_{1}(b, 0) \frac{\partial u_{0}(b)}{\partial x}-a_{2}(b, 0) u_{0}(b) ; \\
\left\|g_{2}\right\|_{C^{((3+\theta) / 2) \alpha}([0,2 \tau])} \\
\leq C\left[\left\|\partial_{t}^{\alpha} u\right\|_{C^{((1+\theta) / 2) \alpha}\left(\Gamma_{2 \tau}\right)}+\left\|u_{0}\right\|_{C^{2+\theta}(\bar{\Omega})}\right] .
\end{gathered}
$$

Due to the fact the functions $f_{0}, f_{2}$, and $u_{0}$ satisfy the consistency conditions, properties (137) allow us to conclude that the right-hand sides (135) meet requirements of Theorem 14. This means that there exists a unique solution $U(x, t) \in$ $C^{2+\theta,((2+\theta) / 2) \alpha}\left(\bar{\Omega}_{T}\right), T=2 \tau$, and that

$$
\begin{gathered}
\|U\|_{C^{2+\theta,((2+\theta) / 2) \alpha}\left(\bar{\Omega}_{T}\right)} \\
\leq C\left[\left\|f_{0}\right\|_{C^{\theta,(\theta / 2) \alpha}\left(\bar{\Omega}_{T}\right)}+\left\|u_{0}\right\|_{C^{2+\theta}(\bar{\Omega})}\right. \\
\left.+\left\|f_{2}\right\|_{C^{((2+\theta) / 2) \alpha}([0, T])}+\left\|\partial_{t}^{\alpha} u\right\|_{C^{((1+\theta) / 2) \alpha}\left(\Gamma_{2 \tau}\right)}\right], \\
\partial_{t}^{\alpha} U=g(t) \quad \text { on } \Gamma_{2 T} ; \\
\left.\partial_{t}^{\alpha} U\right|_{\Gamma_{2 T}} \in C^{((1+\theta) / 2) \alpha}([0, T]) ; \\
U(x, t)=u(x, t) \quad \text { in } \bar{\Omega}_{\tau} .
\end{gathered}
$$

After that, we search the solution of problem (134) in the following form:

$$
\bar{U}(x, t)=U(x, t)+\Theta(x, t),
$$


where the new unknown function $\Theta(x, t)$ satisfies the following conditions:

$$
\begin{aligned}
& \partial_{t}^{\alpha} \Theta(x, t)-a_{0}(x, t) \frac{\partial^{2} \Theta(x, t)}{\partial x^{2}}+a_{1}(x, t) \frac{\partial \Theta(x, t)}{\partial x} \\
& \quad+a_{2}(x, t) \Theta(x, t)=0 \quad \text { in } \Omega_{2 \tau} ; \\
& \partial_{t}^{\alpha} \Theta(b, t)+b_{0}(t) \frac{\partial \Theta(b, t)}{\partial x}+b_{1}(t) \Theta(b, t) \\
& \quad=f_{1}(t)-g(t)-b_{0}(t) \frac{\partial U(b, t)}{\partial x}-b_{1}(t) U(b, t) \\
& \quad \equiv \Phi_{2}(t), \\
& \Theta(a, t)=0, \quad t \in[0,2 \tau] ; \\
& \Theta(x, t)=u(x, t)-U(x, t) \equiv \Phi_{3}(x, t) \quad \text { in } \bar{\Omega}_{\tau} ; \\
& \partial_{t}^{\alpha} \Theta(b, t)=\partial_{t}^{\alpha} u(b, t)-g(t) \equiv \Phi_{4}(t), \quad t \in[0, \tau] .
\end{aligned}
$$

As it follows from (136) and (138), if $t \in[0, \tau]$ then

$$
\begin{gathered}
\Phi_{3}(x, t)=0, \quad x \in \bar{\Omega}, \\
\Phi_{2}(t)=\Phi_{4}(t)=0,
\end{gathered}
$$

$\left\|\Phi_{2}\right\|_{C^{(1+\theta) / 2) \alpha([0,2 \tau])}}$

$$
\begin{aligned}
\leq C & {\left[\left\|f_{0}\right\|_{C^{\theta,(\theta / 2) \alpha}\left(\bar{\Omega}_{2 \tau}\right)}+\left\|u_{0}\right\|_{C^{2+\theta}(\bar{\Omega})}\right.} \\
& \left.+\left\|f_{2}\right\|_{C^{((2+\theta) / 2) \alpha}([0,2 \tau])}+\left\|f_{1}\right\|_{C^{(1+\theta \theta / 2) \alpha}([0,2 \tau])}\right] .
\end{aligned}
$$
Let

In problem (140), we introduce the new variable: $\sigma=t-\tau$.

$$
\begin{gathered}
\bar{\Theta}(x, \sigma):=\Theta(x, \sigma+\tau) ; \\
\bar{a}_{i}(x, \sigma):=a_{i}(x, \sigma+\tau), \quad i=\overline{0,2} \\
\bar{b}_{j}(x, \sigma):=b_{j}(x, \sigma+\tau), \quad j=0,1 ; \\
\bar{\Phi}_{2}(\sigma):=\Phi_{2}(\sigma+\tau),
\end{gathered}
$$

where $\sigma \in[-\tau, \tau]$ and $\overline{a_{i}}, \bar{b}_{j}$ satisfy the conditions of Theorem 3. Due to (141), we have

$$
\begin{gathered}
\bar{\Phi}_{2}(\sigma)=0, \quad \text { if } \sigma \in[-\tau, 0], \\
\bar{\Phi}_{2}(\sigma) \in C^{((1+\theta) / 2) \alpha}([-\tau, \tau]),
\end{gathered}
$$

and therefore

$$
\bar{\Theta}(x, \sigma)=0, \quad \text { if } \sigma \in[-\tau, 0] .
$$

To rewrite problem (140) in the new variable $\sigma$, we use formula (3.110) from [39] as follows:

$$
\partial_{t}^{\alpha} \Theta(x, t)=\partial_{\sigma}^{\alpha} \bar{\Theta}(x, \sigma) .
$$

Thus, problem (140) in the new variable can be rewritten as

$$
\begin{gathered}
\partial_{\sigma}^{\alpha} \bar{\Theta}(x, \sigma)-\bar{a}_{0}(x, \sigma) \frac{\partial^{2} \bar{\Theta}(x, \sigma)}{\partial x^{2}}+\bar{a}_{1}(x, \sigma) \frac{\partial \bar{\Theta}(x, \sigma)}{\partial x} \\
+\bar{a}_{2}(x, \sigma) \bar{\Theta}(x, \sigma)=0 \quad \text { in } \Omega_{\tau} ; \\
\partial_{\sigma}^{\alpha} \bar{\Theta}(b, \sigma)+\bar{b}_{0}(\sigma) \frac{\partial \bar{\Theta}(b, \sigma)}{\partial x}+\bar{b}_{1}(\sigma) \bar{\Theta}(b, \sigma) \\
=\bar{\Phi}_{2}(\sigma), \quad \sigma \in[0, \tau] ; \\
\bar{\Theta}(a, \sigma)=0, \quad \sigma \in[0, \tau] ; \\
\bar{\Theta}(x, 0)=\partial_{\sigma}^{\alpha} \Theta(b, 0)=0 \quad \text { in } \bar{\Omega} .
\end{gathered}
$$

Then we apply Lemma 15 to problem (147) and get the one-to-one solvability in $C^{2+\Theta,((2+\Theta) / 2) \alpha}\left(\bar{\Omega}_{\tau}\right)$, and the estimate like (123) holds. Returning to the old variable $t=$ $\sigma+\tau$, we obtain the unique solution of problem (140), $\Theta(x, t) \in C^{2+\theta,((2+\theta) / 2) \alpha}\left(\bar{\Omega}_{2 \tau}\right)$, and $\Theta(x, t)$ satisfies inequality (123), where $\tau:=2 \tau$.

Thus, representation (139) together with the properties (138) for the functions $U(x, t)$ and the corresponding properties of $\Theta(x, t)$ allow us to construct the solution of problem (1)-(5) for $t \in[\tau, 2 \tau]$, if there is the solution for $t \in$ $[0, \tau]$. Repeating this procedure, we prove the existence of the solution for $t \in[0, T]$ and obtain estimate (19). As for the uniqueness of the solution, it follows from inequality (19). This ends the proof of Theorem 3.

\section{The Local Solvability of the Nonlinear Problem with a Fractional Dynamic Boundary Condition}

In this section, we indicate how our results may be applied to the nonlinear problem:

$$
\begin{aligned}
& \partial_{t}^{\alpha} v-a_{0}\left(v, v_{x}\right) v_{x x}=a_{1}\left(v, v_{x}\right), \\
& (x, t) \in \Omega_{T}, \quad \alpha \in(0,1) ; \\
& v(x, 0)=u_{0}(x) \text { in } \Omega ; \\
& \partial_{t}^{\alpha} v-b_{0}^{i}(v) v_{x}=b_{1}^{j}(v) \quad \text { on } \Gamma_{j T}, j=1,2 .
\end{aligned}
$$

We require the following conditions on the functions $a_{i}, b_{k}^{j}$, $k, i=0,1 ; j=1,2$ :

(i) There exist some positive constants $\delta_{l}, l=\overline{0,7}$ such that

$$
\begin{gathered}
0<\delta_{0}<a_{0}\left(v, v_{x}\right)<\delta_{1} ; \quad 0<\delta_{2}<b_{0}^{1}(v)<\delta_{3} ; \\
-\delta_{4}<b_{0}^{2}(v)<-\delta_{5}<0 ; \\
\left|b_{1}^{j}\right|<\delta_{6}, \quad j=1,2 ; \\
\left|a_{1}\left(v, v_{x}\right)\right| \leq \delta_{7} .
\end{gathered}
$$


(ii) Consistency conditions hold:

$$
\begin{aligned}
& a_{0}\left(u_{0}, u_{0 x}\right) u_{0 x x}+a_{1}\left(u_{0}, u_{0 x}\right) \\
& \quad=b_{0}^{j}\left(u_{0}\right) u_{0 x}+b_{1}^{j}\left(u_{0}\right) \text { on } \Gamma_{j T}, j=1,2 .
\end{aligned}
$$

(iii) There exist $\partial a_{i}\left(v, v_{x}\right) / \partial v, \partial a_{i}\left(v, v_{x}\right) / \partial v_{x}, \partial b_{k}^{j}(v) / \partial v$, $k, i=0,1, j=1,2$, such that for some positive constant $L$

$\sum_{i=0}^{1}\left[\left|\frac{\partial a_{i}\left(v, v_{x}\right)}{\partial v}-\frac{\partial a_{i}\left(u, u_{x}\right)}{\partial u}\right|+\left|\frac{\partial a_{i}\left(v, v_{x}\right)}{\partial v_{x}}-\frac{\partial a_{i}\left(u, u_{x}\right)}{\partial u_{x}}\right|\right]$ $\leq L\left[|v-u|+\left|v_{x}-u_{x}\right|\right]$

$\sum_{j=1}^{2}\left|\frac{\partial b_{0}^{j}(v)}{\partial v}-\frac{\partial b_{0}^{j}(u)}{\partial u}\right|+\left|\frac{\partial b_{1}^{j}(v)}{\partial v}-\frac{\partial b_{1}^{j}(u)}{\partial u}\right|$

$\leq L|v-u|$

for any bounded functions $u, v$ together with their derivatives.

(iv) There are positive constants $A_{i}, B_{j}, i, j=\overline{0,3}$ as follows:

$$
\begin{array}{rlrl}
\left|\frac{\partial a_{0}\left(v, v_{x}\right)}{\partial v}\right| \leq A_{0} ; & & \left|\frac{\partial a_{1}\left(v, v_{x}\right)}{\partial v}\right| \leq A_{1} ; \\
\left|\frac{\partial a_{0}\left(v, v_{x}\right)}{\partial v_{x}}\right| \leq A_{2} ; & \left|\frac{\partial a_{1}\left(v, v_{x}\right)}{\partial v_{x}}\right| \leq A_{3} ; \\
\left|\frac{\partial b_{0}^{1}(v)}{\partial v}\right| \leq B_{0} ; & \left|\frac{\partial b_{0}^{2}(v)}{\partial v}\right| \leq B_{1} ; \\
\left|\frac{\partial b_{1}^{1}(v)}{\partial v}\right| \leq B_{2} ; & \left|\frac{\partial b_{1}^{2}(v)}{\partial v}\right| \leq B_{3} ;
\end{array}
$$

(v)

$$
u_{0}(x) \in C^{2+\theta}(\bar{\Omega})
$$

We introduce the functional spaces $\mathbb{U}$ and $\mathbb{M}_{0}$ as follows:

$$
\begin{gathered}
\mathbb{W}=C^{2+\theta,((2+\theta) / 2) \alpha}\left(\bar{\Omega}_{T}\right) \times C^{((1+\theta) / 2) \alpha}\left(\Gamma_{1 T}\right) \times C^{((1+\theta) / 2) \alpha}\left(\Gamma_{2 T}\right) \\
\mathbb{T}_{0}=C^{\theta,(\theta / 2) \alpha}\left(\bar{\Omega}_{T}\right) \times C^{((1+\theta) / 2) \alpha}\left(\Gamma_{1 T}\right) \times C^{((1+\theta) / 2) \alpha}\left(\Gamma_{2 T}\right),
\end{gathered}
$$

and for any elements $z=\left(v,\left.\partial_{t}^{\alpha} v\right|_{\partial \Omega_{T}}\right) \in \mathbb{H}$, and $\mathfrak{F}(z)=$ $\left(\Phi_{0}, \Phi_{1}, \Phi_{2}\right) \in \mathbb{H}_{0}$, we denote

$$
\begin{gathered}
\|z\|_{\mathbb{\boxplus}}:=\|v\|_{C^{2+\theta,((2+\theta) / 2) \alpha}\left(\bar{\Omega}_{T}\right)}+\left\|\partial_{t}^{\alpha} v\right\|_{C^{((1+\theta) / 2) \alpha}\left(\Gamma_{1 T}\right)} \\
+\left\|\partial_{t}^{\alpha} v\right\|_{C^{((1+\theta) / 2) \alpha}\left(\Gamma_{2 T}\right)} ; \\
\|\mathfrak{F}(z)\|_{\mathbb{H}_{0}}:=\left\|\Phi_{0}\right\|_{C^{\theta,(\theta / 2) \alpha}\left(\bar{\Omega}_{T}\right)}+\left\|\Phi_{1}\right\|_{C^{((1+\theta) / 2) \alpha}\left(\Gamma_{1 T}\right)} \\
+\left\|\Phi_{2}\right\|_{C^{((1+\theta) / 2) \alpha}\left(\Gamma_{2 T}\right)} .
\end{gathered}
$$

Let $B_{r}(v)=\left\{v \in \mathbb{H}:\|v\|_{\mathbb{Q}} \leq r\right\}$ and $B_{r}^{0}(v)=\{v \in$ $\left.\mathbb{W}:\|v\|_{\mathbb{Q}} \leq r, v(x, 0)=0\right\}, r<r_{0}$, be balls of radius $r$ in the space $\mathbb{H}$, centered at the origin, for some positive $r_{0}$ to be determined later on.

The simple calculations lead to the following assertion.

Proposition 16. Let conditions (149)-(153) hold and the function $u \in B_{r}(u)$; then $a_{i}\left(u, u_{x}\right), \partial a_{i}\left(u, u_{x}\right) / \partial u, \partial a_{i}\left(u, u_{x}\right) / \partial u_{x} \in$ $C^{\theta,(\theta / 2)}\left(\bar{\Omega}_{T}\right) ; b_{k}^{j}(u), \partial b_{k}^{j} / \partial u \in C^{((1+\theta) / 2)}\left(\Gamma_{j T}\right) ; i, k=0,1, j=$ 1,2 , and

$\left\|a_{0}\right\|_{C^{\theta,(\theta / 2) \alpha}\left(\bar{\Omega}_{T}\right)}$

$$
\leq \max \left\{\delta_{0}, \delta_{1}\right\}+\left[A_{0}+A_{2}\right]\|u\|_{\mathbb{\square}}\left[1+T^{\alpha / 2}+T^{((2-\theta) / 2) \alpha}\right] ;
$$

$\left\|a_{1}\right\|_{C^{\theta,(\theta / 2) \alpha}\left(\bar{\Omega}_{T}\right)}$

$$
\leq \delta_{7}+\left[A_{1}+A_{3}\right]\|u\|_{\mathbb{G}}\left[1+T^{\alpha / 2}+T^{((2-\theta) / 2) \alpha}\right] ;
$$

$\sum_{j=1}^{2}\left\|b_{0}^{j}\right\|_{C^{((1+\theta) / 2) \alpha}\left(\Gamma_{j T}\right)}$

$$
\begin{aligned}
\leq & \max \left\{\delta_{2}, \delta_{3}\right\}+\max \left\{\delta_{4}, \delta_{5}\right\} \\
& +\left[B_{0}+B_{1}\right]\|u\|_{\mathbb{G}}\left[T^{\alpha}+T^{((1+\theta) / 2) \alpha}\right] ;
\end{aligned}
$$

$\left\|b_{1}^{1}\right\|_{C^{(1+\theta) / 2) \alpha}\left(\Gamma_{1 T}\right)}+\left\|b_{1}^{2}\right\|_{C^{((1+\theta) / 2) \alpha}\left(\Gamma_{2 T}\right)}$

$$
\leq \delta_{6}+\left[B_{3}+B_{2}\right]\|u\|_{\mathbb{Q}}\left[T^{\alpha}+T^{((1+\theta) / 2) \alpha}\right] ;
$$

$$
\begin{aligned}
& \left\|\frac{\partial a_{0}}{\partial u}\right\|_{C^{\theta,(\theta / 2) \alpha}\left(\bar{\Omega}_{T}\right)}+\left\|\frac{\partial a_{0}}{\partial u_{x}}\right\|_{C^{\theta,(\theta / 2) \alpha}\left(\bar{\Omega}_{T}\right)} \\
& \quad \leq 2 L\|u\|_{\mathbb{H}}\left[1+T^{((2-\theta) / 2) \alpha}+T^{\alpha / 2}\right]+A_{0}+A_{2} ;
\end{aligned}
$$

$\left\|\frac{\partial a_{1}}{\partial u}\right\|_{C^{\theta,(\theta / 2) \alpha}\left(\bar{\Omega}_{T}\right)}+\left\|\frac{\partial a_{1}}{\partial u_{x}}\right\|_{C^{\theta,(\theta / 2) \alpha}\left(\bar{\Omega}_{T}\right)}$

$$
\leq 2 L\|u\|_{\mathbb{\square}}\left[1+T^{((2-\theta) / 2) \alpha}+T^{\alpha / 2}\right]+A_{1}+A_{3} ;
$$

$$
\begin{aligned}
\| \frac{\partial b_{0}^{1}}{\partial u} & \left\|_{C^{(1+\theta) / 2) \alpha}\left(\Gamma_{1 T}\right)}+\right\| \frac{\partial b_{0}^{2}}{\partial u} \|_{C^{((1+\theta) / 2) \alpha}\left(\Gamma_{2 T}\right)} \\
& \leq 2 L\|u\|_{\mathbb{G}}\left[1+T^{((1-\theta) / 2) \alpha}\right]+B_{0}+B_{1} ;
\end{aligned}
$$

$$
\begin{aligned}
\left\|\frac{\partial b_{1}^{1}}{\partial u}\right\|_{C^{(1+\theta) / 2) \alpha}\left(\Gamma_{1 T}\right)}+\left\|\frac{\partial b_{1}^{2}}{\partial u}\right\|_{C^{((1+\theta) / 2) \alpha}\left(\Gamma_{2 T}\right)} \\
\leq 2 L\|u\|_{\mathbb{Q}}\left[1+T^{((1-\theta) / 2) \alpha}\right]+B_{3}+B_{2} .
\end{aligned}
$$

Moreover, for the functions $p_{\sigma}(u, v):=\sigma u+(1-\sigma) v$ and $q_{\sigma}(u, v):=\sigma u_{x}+(1-\sigma) v_{x}$, for all $\sigma \in[0,1], u, v \in B_{r}(u)$, the following inequalities hold:

$$
\begin{aligned}
& \left|\frac{\partial a_{i}\left(p_{\sigma}(u, v), v_{x}\right)}{\partial p_{\sigma}(u, v)}\right| \leq L \max _{\bar{\Omega}_{T}}|u|+\max _{x, t, v, v_{x}}\left|\frac{\partial a_{i}\left(v, v_{x}\right)}{\partial v}\right| ; \\
& \left|\frac{\partial a_{i}\left(v, q_{\sigma}(u, v)\right)}{\partial q_{\sigma}(u, v)}\right| \leq L \max _{\bar{\Omega}_{T}}\left|u_{x}\right|+\max _{x, t, v, v_{x}}\left|\frac{\partial a_{i}\left(v, v_{x}\right)}{\partial v_{x}}\right| ;
\end{aligned}
$$




$$
\begin{array}{r}
\left|\frac{\partial b_{i}^{j}\left(p_{\sigma}(u, v)\right)}{\partial p_{\sigma}(u, v)}\right| \leq L \max _{\bar{\Omega}_{T}}|u|+\max _{x, t, v}\left|\frac{\partial b_{i}^{j}(v)}{\partial v}\right|, \\
i=0,1 ; j=1,2 .
\end{array}
$$

First, we linearize problem (148) on the initial data and represent one as a system $\mathfrak{A} z=\mathfrak{I}(z)$, where $\mathfrak{A}$ is a linear operator and $\mathfrak{I}(z)$ is a nonlinear perturbation. To this end, we introduce the new unknown function as follows:

$$
w(x, t)=u(x, t)-V_{0}(x, t),
$$

where the function $V_{0}(x, t)$ is a solution of the following problem:

$$
\begin{gathered}
\partial_{t}^{\alpha} V_{0}-a_{0}\left(u_{0}, u_{0 x}\right) V_{0 x x}=a_{1}\left(u_{0}, u_{0 x}\right), \\
(x, t) \in \Omega_{T}, \alpha \in(0,1) ; \\
V(x, 0)=u_{0}(x) \quad \text { in } \Omega ; \\
\partial_{t}^{\alpha} V_{0}-b_{0}^{i}\left(u_{0}\right) V_{0 x}=b_{1}^{j}\left(u_{0}\right) \quad \text { on } \Gamma_{j T}, \quad j=1,2 .
\end{gathered}
$$

By Theorem 5 and Proposition 16, there exists a unique solution $V_{0}(x, t)$ of the problem (159) and

$$
\left\|V_{0}\right\|_{\mathbb{\sharp}} \leq C_{0}\left\|u_{0}\right\|_{C^{2+\theta}(\bar{\Omega})},
$$

where the positive constant $C_{0}$ depends on $\delta_{i}, i=\overline{0,7}, B_{k}, A_{j}$, $k, j=\overline{0,3}, L$.

Next, we rewrite problem (148) in terms of the function $w(x, t)$ and after some calculations get the problem in the form:

$$
\begin{gathered}
\partial_{t}^{\alpha} w-a_{0}\left(V_{0}, V_{0 x}\right) w_{x x}=\Phi_{0}\left(w, w_{x}, w_{x x}\right), \\
(x, t) \in \Omega_{T}, \alpha \in(0,1) ; \\
w(x, 0)=0 \quad \text { in } \Omega ; \\
\partial_{t}^{\alpha} w-b_{0}^{i}\left(V_{0}\right) w_{x}=\Phi_{1}^{j}\left(w, w_{x}\right) \quad \text { on } \Gamma_{j T}, j=1,2,
\end{gathered}
$$

where

$$
\begin{aligned}
\Phi_{0}\left(w, w_{x}, w_{x x}\right) & \\
= & f_{0}(x, t)+\left[a_{0}\left(V_{0}+w, V_{0 x}+w_{x}\right)-a_{0}\left(V_{0}, V_{0 x}\right)\right] \\
\times & {\left[w_{x x}+V_{0 x x}\right] } \\
+ & {\left[a_{1}\left(V_{0}+w, V_{0 x}+w_{x}\right)-a_{1}\left(V_{0}, V_{0 x}\right)\right] } \\
f_{0}(x, t)= & {\left[a_{0}\left(V_{0}, V_{0 x}\right)-a_{0}\left(u_{0}, u_{0 x}\right)\right] V_{0 x x} } \\
& +\left[a_{1}\left(V_{0}, V_{0 x}\right)-a_{1}\left(u_{0}, u_{0 x}\right)\right] \\
\Phi_{1}^{j}\left(w, w_{x}\right)= & f_{1}^{j}(x, t) \\
& +\left[b_{0}^{j}\left(V_{0}+w\right)-b_{0}^{j}\left(V_{0}\right)\right]\left[w_{x}+V_{0 x}\right] \\
& +\left[b_{1}^{j}\left(V_{0}+w\right)-b_{1}^{j}\left(V_{0}\right)\right], \\
f_{1}^{j}(x, t)=\left[b_{0}^{j}\right. & \left.\left(V_{0}\right)-b_{0}^{j}\left(u_{0}\right)\right] V_{0 x}+\left[b_{1}^{j}\left(V_{0}\right)-b_{1}^{j}\left(u_{0}\right)\right] .
\end{aligned}
$$

Thus, we represent nonlinear problem (148) as

$$
\mathfrak{A} w=\mathfrak{I}(w) .
$$

Note that if we froze the functional arguments in the functions $\Phi_{0}\left(w, w_{x}, w_{x x}\right)$ and $\Phi_{1}^{j}\left(w, w_{x}\right), j=1,2$, then problem (161) will be a linear problem with variable coefficients, which has been studied in detail in Sections 2-4. By Theorem 5, $\mathfrak{A}$ has a bounded inverse $\mathfrak{A}^{-1}$, so that

$$
w=\mathfrak{A}^{-1} \mathfrak{T}(w) \equiv \mathfrak{S}(w),
$$

and $\mathfrak{S}$ is a nonlinear operator. We will show that $\mathfrak{S}$ is a contraction operator.

Lemma 17. The following inequalities hold for the right-hand sides of problem (167):

$$
\begin{array}{r}
\|\mathfrak{I}(0)\|_{\mathbb{Q}_{0}} \leq C_{1}(T), \\
\|\mathfrak{I}(w)-\mathfrak{I}(v)\|_{\mathbb{Q}_{0}} \leq C_{2}(T, r)\|w-v\|_{\mathbb{q}}, \\
\forall w, v, \in B_{r}^{0}(u),
\end{array}
$$

with $C_{1}(T) \rightarrow 0 C_{2}(T, r) \rightarrow 0$ as $r, T \rightarrow 0$.

Proof. First we prove (168). Note that

$$
\|\mathfrak{I}(0)\|_{\mathbb{\sharp}_{0}}=\left\|f_{0}\right\|_{C^{\theta,(\theta / 2) \alpha}\left(\bar{\Omega}_{T}\right)}+\sum_{j=1}^{2}\left\|f_{1}^{j}\right\|_{C^{((1+\theta) / 2) \alpha}\left(\Gamma_{j T}\right)} .
$$

Let

$$
\begin{gathered}
p_{\sigma}:=p_{\sigma}\left(z_{1}, z_{2}\right)=\sigma z_{1}(x, t)+(1-\sigma) z_{2}(x, t) ; \\
q_{\sigma}:=q_{\sigma}\left(z_{1}, z_{2}\right) \\
=\sigma z_{1 x}(x, t)+(1-\sigma) z_{2 x}(x, t) ; \\
\forall z_{i}(x, t) \in B_{r}(u), i=1,2, \sigma \in[0,1] .
\end{gathered}
$$

Using definitions (163), (165) and notations (171) with $z_{1}:=$ $V_{0}(x, t)$ and $z_{2}:=u_{0}(x)$, we have

$$
\begin{aligned}
f_{0}(x, t)= & V_{0 x x} \int_{0}^{1} \frac{\partial a_{0}\left(p_{\sigma}, V_{0 x}\right)}{\partial p_{\sigma}} d \sigma\left[V_{0}(x, t)-u_{0}(x)\right] \\
& +V_{0 x x} \int_{0}^{1} \frac{\partial a_{0}\left(u_{0}, q_{\sigma}\right)}{\partial q_{\sigma}} d \sigma\left[V_{0 x}(x, t)-u_{0 x}(x)\right] \\
& +\int_{0}^{1} \frac{\partial a_{1}\left(p_{\sigma}, V_{0 x}\right)}{\partial p_{\sigma}} d \sigma\left[V_{0}(x, t)-u_{0}(x)\right] \\
& +\int_{0}^{1} \frac{\partial a_{1}\left(u_{0}, q_{\sigma}\right)}{\partial q_{\sigma}} d \sigma\left[V_{0 x}(x, t)-u_{0 x}(x)\right] \\
f_{1}^{j}(x, t)= & V_{0 x} \int_{0}^{1} \frac{\partial b_{0}^{j}\left(p_{\sigma}\right)}{\partial p_{\sigma}} d \sigma\left[V_{0}(x, t)-u_{0}(x)\right] \\
& +\int_{0}^{1} \frac{\partial b_{1}^{j}\left(p_{\sigma}\right)}{\partial p_{\sigma}} d \sigma\left[V_{0}(x, t)-u_{0}(x)\right] .
\end{aligned}
$$


It is easy to examine that the following are true:

$$
\begin{gathered}
\left|V_{0}(x, t)-u_{0}(x)\right| \leq T^{\alpha}\left\|V_{0}\right\|_{\mathbb{\sharp}} ; \\
\left|V_{0 x}(x, t)-u_{0 x}(x)\right| \leq T^{((1+\theta) / 2) \alpha}\left\|V_{0}\right\|_{\mathbb{\boxplus}} ; \\
\left\langle V_{0}-u_{0}\right\rangle_{x, \Omega_{T}}^{(\theta)} \leq T^{((1+\theta) / 2) \alpha}\left\|V_{0}\right\|_{\mathbb{\sharp} ;} \\
\left\langle V_{0 x}-u_{0 x}\right\rangle_{x, \Omega_{T}}^{(\theta)} \leq T^{(\theta / 2) \alpha}\left\|V_{0}\right\|_{\mathbb{A}} ; \\
\left\langle V_{0}-u_{0}\right\rangle_{t, \Omega_{T}}^{((\theta / 2) \alpha)} \leq T^{((2-\theta) / 2) \alpha}\left\|V_{0}\right\|_{\mathbb{\boxplus}} ; \\
\left\langle V_{0 x}-u_{0 x}\right\rangle_{t, \Omega_{T}}^{((\theta / 2) \alpha)} \leq T^{\alpha / 2}\left\|V_{0}\right\|_{\mathbb{H}} .
\end{gathered}
$$

Hence, using inequalities (173), representation (172), and results of Proposition 16, we get after some tedious calculations

$$
\begin{aligned}
& \left\|f_{0}\right\|_{C^{\theta,(\theta / 2) \alpha}\left(\bar{\Omega}_{T}\right)} \\
& \leq C_{1} T^{\theta \alpha / 2}\left[1+\sum_{i=0}^{3} A_{i}\right] \\
& \times\left[1+L\left\|u_{0}\right\|_{C^{2+\theta}(\bar{\Omega})}\left(1+T^{\alpha / 2}+T^{(2-\theta) \alpha / 2}\right)\right]^{2} ; \\
& \sum_{j=1}^{2}\left\|f_{1}^{j}\right\|_{C^{(1+\theta) / 2) \alpha}\left(\Gamma_{j T}\right)} \\
& \leq C_{1} \max \left\{T^{\theta / \alpha 2}, T^{(1-\theta) \alpha / 2}\right\}\left[1+\sum_{i=0}^{3} B_{i}\right] \\
& \times\left[1+L\left\|u_{0}\right\|_{C^{2+\theta}(\bar{\Omega})}\left(1+T^{(1-\theta) \alpha / 2}\right)\right]^{2}, \\
& \sqrt{C_{1}}=\max \left\{1, C_{0}\right\},
\end{aligned}
$$

where the constant $C_{0}$ is from (160).

Let

$$
\begin{aligned}
C_{1}(T):= & C_{1} \max \left\{T^{\theta / \alpha 2}, T^{(1-\theta) \alpha / 2}\right\} \\
& \times\left[1+\sum_{i=0}^{3}\left(B_{i}+A_{i}\right)\right] \\
& \times\left[1+L\left\|u_{0}\right\|_{C^{2+\theta}(\bar{\Omega})}\left(1+T^{\theta / \alpha 2}+T^{(2-\theta) \alpha / 2}\right)\right]^{2} .
\end{aligned}
$$

It is easy to see that $C_{1}(T) \rightarrow 0$, as $T \rightarrow 0$. Thus inequalities (174) together with representation (170) lead to estimate (168), where $C_{1}(T)$ is given by (175).
Next, we will obtain inequality (169). Note that, as it follows from definitions (162) and (164), one has

$$
\begin{aligned}
\| \mathfrak{T}(w)- & \mathfrak{T}(v) \|_{\mathbb{q}_{0}} \\
= & \|\left[\Phi_{0}\left(w, w_{x}, w_{x x}\right)-f_{0}\right] \\
& -\left[\Phi_{0}\left(v, v_{x}, v_{x x}\right)-f_{0}\right] \|_{C^{\theta,(\theta / 2) \alpha}\left(\bar{\Omega}_{T}\right)} \\
+ & \sum_{j=1}^{2} \|\left[\Phi_{1}^{j}\left(w, w_{x}\right)-f_{1}^{j}\right] \\
& -\left[\Phi_{1}^{j}\left(v, v_{x}\right)-f_{1}^{j}\right] \|_{C^{((1+\theta) / 2) \alpha}\left(\Gamma_{j T}\right)} .
\end{aligned}
$$

\section{Denote}

$$
\begin{gathered}
l_{\sigma}=\sigma w_{x x}(x, t)+(1-\sigma) v_{x x}(x, t), \quad \sigma \in[0,1] ; \\
Q\left(z, z_{x}, z_{x x}\right) \\
:=\left[a_{0}\left(\mathrm{~V}_{0}+z, V_{0 x}+z_{x}\right)-a_{0}\left(V_{0}, V_{0 x}\right)\right]\left[z_{x x}+V_{0 x x}\right] ; \\
Q_{1}^{j}\left(z, z_{x}\right):=\left[b_{0}^{j}\left(V_{0}+z\right)-b_{0}^{j}\left(V_{0}\right)\right]\left[z_{x}+V_{0 x}\right], \\
\forall z \in B_{r}^{0}(u) .
\end{gathered}
$$

Then, we have the following:

$$
\begin{aligned}
& {\left[\Phi_{0}\left(w, w_{x}, w_{x x}\right)-f_{0}\right]-\left[\Phi_{0}\left(v, v_{x}, v_{x x}\right)-f_{0}\right]} \\
& =\int_{0}^{1} \frac{\partial Q\left(p_{\sigma}, w_{x}, w_{x x}\right)}{\partial p_{\sigma}} d \sigma(w-v) \\
& \quad+\int_{0}^{1} \frac{\partial Q\left(v, q_{\sigma}, w_{x x}\right)}{\partial q_{\sigma}} d \sigma\left(w_{x}-v_{x}\right) \\
& \quad+\int_{0}^{1} \frac{\partial Q\left(v, v_{x}, l_{\sigma}\right)}{\partial l_{\sigma}} d \sigma\left(w_{x x}-v_{x x}\right)+(w-v) \\
& \quad \times \int_{0}^{1} \frac{\partial a_{1}\left(p_{\sigma}, V_{0 x}+w_{x}\right)}{\partial p_{\sigma}} d \sigma \\
& \quad+\int_{0}^{1} \frac{\partial a_{1}\left(V_{0}+v, q_{\sigma}\right)}{\partial q_{\sigma}} d \sigma\left(w_{x}-v_{x}\right) ; \\
& \quad\left[\Phi_{1}^{j}\left(w, w_{x}\right)-f_{1}^{j}\right]-\left[\Phi_{1}^{j}\left(v, v_{x}\right)-f_{1}^{j}\right] \\
& \quad=\int_{0}^{1} \frac{\partial Q_{1}^{j}\left(p_{\sigma}, w_{x}\right)}{\partial p_{\sigma}} d \sigma(w-v) \\
& \quad+\int_{0}^{1} \frac{\partial Q_{1}^{j}\left(v, q_{\sigma}\right)}{\partial q_{\sigma}} d \sigma\left(w_{x}-v_{x}\right) \\
& \quad \\
& \quad b_{1}^{j}\left(p_{\sigma}\right) \\
& \quad d \sigma(w-v) .
\end{aligned}
$$


After that, the simple calculations together with the results of Proposition 16 allow us to get the following for any function $z(x, t) \in B_{r}^{0}(u)$.

(i)

$\left\|\frac{\partial Q\left(z, z_{x}, z_{x x}\right)}{\partial z}\right\|_{C^{\theta,(\theta / 2) \alpha}\left(\bar{\Omega}_{T}\right)}$

$$
\begin{aligned}
& +\left\|\frac{\partial Q\left(z, z_{x}, z_{x x}\right)}{\partial z_{x}}\right\|_{C^{\theta,(\theta / 2) \alpha}\left(\bar{\Omega}_{T}\right)} \\
& +\left\|\frac{\partial Q_{1}^{1}\left(z, z_{x}\right)}{\partial z}\right\|_{C^{(1+\theta) / 2) \alpha}\left(\Gamma_{1 T}\right)} \\
& +\left\|\frac{\partial Q_{1}^{2}\left(z, z_{x}\right)}{\partial z}\right\|_{C^{(1+\theta) / 2) \alpha}\left(\Gamma_{2 T}\right)} \\
& \leq\left[\|z\|_{\mathbb{W}}+C_{0}\left\|u_{0}\right\|_{C^{2+\theta}(\bar{\Omega})}\right] \\
& \times\left[2 L\|z\|_{\mapsto \varpi}+\sum_{j=0}^{1} B_{j}+A_{0}+A_{2}\right] .
\end{aligned}
$$

(ii) $\max _{\bar{\Omega}_{T}}\left|\frac{\partial Q\left(z, z_{x}, z_{x x}\right)}{\partial z_{x x}}\right|$

$$
\leq T^{(1+\theta) \alpha / 2}\|z\|_{\mathbb{H}}\left[2 L\|z\|_{\mathbb{H}}+A_{0}+A_{2}\right]\left[1+T^{\alpha / 2}\right] ;
$$

$$
\begin{aligned}
& \left\langle\frac{\partial Q\left(z, z_{x}, z_{x x}\right)}{\partial z_{x x}}\right\rangle_{t, \Omega_{T}}^{((\theta / 2) \alpha)} \\
& \leq T^{\alpha / 2}\|z\|_{\mathbb{H}}\left[2 L\|z\|_{\mathbb{H}}+A_{0}+A_{2}\right] \\
& \times\left[1+T^{\theta \alpha / 2}+T^{(1-\theta) \alpha / 2}\right] ; \\
& \left\langle\frac{\partial Q\left(z, z_{x}, z_{x x}\right)}{\partial z_{x x}}\right\rangle_{x, \Omega_{T}}^{(\theta)} \\
& \leq T^{(1+\theta) \alpha / 2}\|z\|_{\text {↔ }}\left[2 L\|z\|_{\text {↔๑ }}+A_{0}+A_{2}\right] \\
& \times\left[1+T^{(1-\theta) \alpha / 2}\right] .
\end{aligned}
$$

$$
\begin{aligned}
& \text { (iii) } \max _{\bar{\Omega}_{T}}\left|\frac{\partial Q_{1}^{j}\left(z, z_{x}\right)}{\partial z_{x}}\right| \leq T^{\alpha}\|z\|_{\mathbb{H}}\left[L\|z\|_{\mathbb{\uplus}}+\sum_{j=0}^{1} B_{j}\right] ; \\
& \left\langle\frac{\partial Q_{1}^{j}\left(z, z_{x}\right)}{\partial z_{x}}\right\rangle_{t, \Omega_{T}}^{(((1+\theta) / 2) \alpha)} \\
& \leq T^{(1-\theta) \alpha / 2}\left[1+T^{(1+\theta) \alpha / 2}\right]\left[L\|z\|_{\mathbb{H}}+\sum_{j=0}^{1} B_{j}\right] ;
\end{aligned}
$$

$$
\begin{aligned}
& \left\langle\frac{\partial Q_{1}^{j}\left(z, z_{x}\right)}{\partial z_{x}}\right\rangle_{x, \Omega_{T}}^{(\theta)} \\
& \leq T^{(1+\theta) \alpha / 2}\|z\|_{\mathbb{\boxplus}}\left[L\|z\|_{\mathbb{Q}}+\sum_{j=0}^{1} B_{j}\right]\left[1+T^{(1-\theta) \alpha / 2}\right] .
\end{aligned}
$$

Moreover, due to $v, w \in B_{r}^{0}(u)$, we have the following:

$$
\begin{gathered}
T^{-\alpha} \max _{\bar{\Omega}_{T}}|v-w|+T^{-(1+\theta) \alpha / 2} \max _{\bar{\Omega}_{T}}\left|v_{x}-w_{x}\right| \\
+T^{-\theta \alpha / 2} \max _{\bar{\Omega}_{T}}\left|v_{x x}-w_{x x}\right| \leq\|w-v\|_{\mathbb{\sharp}} ; \\
T^{-(1+\theta) \alpha / 2}\langle v-w\rangle_{x, \Omega_{T}}^{(\theta)}+T^{-\theta \alpha / 2}\left\langle v_{x}-w_{x}\right\rangle_{x, \Omega_{T}}^{(\theta)} \\
\leq\|w-v\|_{\mathbb{\sharp}} ; \\
T^{-((2-\theta) / 2) \alpha}\langle v-w\rangle_{t, \Omega_{T}}^{((\theta / 2) \alpha)}+T^{-\alpha / 2}\left\langle v_{x}-w_{x}\right\rangle_{t, \Omega_{T}}^{((\theta / 2) \alpha)} \\
\leq\|w-v\|_{\mathbb{H}} .
\end{gathered}
$$

Thus, applying inequalities from Proposition 16 and (179)(182) to the right-hand sides in (178), we get (169), where

$$
\begin{aligned}
C_{2}(T, r)= & \sqrt{C_{1}} \max \left\{T^{\theta \alpha / 2}, T^{(1-\theta) \alpha / 2}\right\} \\
& \times\left[L r+\sum_{i=0}^{3}\left(B_{i}+A_{i}\right)\right]\left[1+r+\left\|u_{0}\right\|_{C^{2+\theta}(\bar{\Omega})}\right] \\
& \times\left[1+T^{3 \alpha / 2}+T^{(3+\theta) \alpha / 2}+T^{\alpha+\alpha \theta}\right] .
\end{aligned}
$$

It is obviously $C_{2}(T, r) \rightarrow 0$ as $r, T \rightarrow 0$.

As it follows from Lemma 17, for sufficiently small $T$ and $r_{0}$, the nonlinear operator $\mathfrak{S}$ satisfies the conditions of the fixed point theorem for a contraction operator. Hence, we have proved the following theorem.

Theorem 18. Let conditions (149)-(153) hold. Then there exists a unique solution of problem (148) for small interval $0<$ $t<T$, such that $v \in C^{2+\theta,((2+\theta) / 2) \alpha}\left(\bar{\Omega}_{T}\right)$ and that $\partial_{t}^{\alpha} v \in$ $C^{((1+\theta) / 2) \alpha}\left(\Gamma_{j T}\right), j=1,2$.

Using the analogous arguments, it is possible to assert the following results.

Remark 19. If we exchange the dynamic boundary condition on $\Gamma_{1 T}$ by either (5) or (6) and assume that the corresponding consistency conditions hold, then the results of Theorem 18 save. 
Remark 20. The results of Theorem 18 hold if conditions (152) are changed by

$$
\begin{gathered}
\left|\frac{\partial a_{0}\left(v, v_{x}\right)}{\partial v}\right| \leq A_{0}\left[1+|v|+\left|v_{x}\right|\right] ; \\
\left|\frac{\partial a_{1}\left(v, v_{x}\right)}{\partial v}\right| \leq A_{1}\left[1+|v|+\left|v_{x}\right|\right] ; \\
\left|\frac{\partial a_{0}\left(v, v_{x}\right)}{\partial v_{x}}\right| \leq A_{2}\left[1+|v|+\left|v_{x}\right|\right] ; \\
\left|\frac{\partial a_{1}\left(v, v_{x}\right)}{\partial v_{x}}\right| \leq A_{3}\left[1+|v|+\left|v_{x}\right|\right] ; \\
\left|\frac{\partial b_{0}^{1}(v)}{\partial v}\right| \leq B_{0}[1+|v|] ; \quad\left|\frac{\partial b_{0}^{2}(v)}{\partial v}\right| \leq B_{1}[1+|v|] ; \\
\left|\frac{\partial b_{1}^{1}(v)}{\partial v}\right| \leq B_{2}[1+|v|] ; \quad\left|\frac{\partial b_{1}^{2}(v)}{\partial v}\right| \leq B_{3}[1+|v|]
\end{gathered}
$$

and constants $\delta_{6}$ and $\delta_{7}$ in requirements (149) are replaced by $\delta_{6}[1+|v|]$ and $\delta_{7}\left[1+|v|+\left|v_{x}\right|\right]$, correspondingly.

\section{Appendix}

Here we give the proof of some estimates used in the arguments above.

\section{A. The proof of Lemma 6}

The statement (i) and the first inequality in (iii) of Lemma 6 follow immediately from representation (72) of the function $G(y, t)$ and the next properties of the Wright functions:

$$
0<W(-z ;-\beta, \gamma) \leq C \exp \left(-\kappa z^{(1 /(1-\beta))}\right) \quad \text { if } \gamma, z \geq 0
$$

where the constant $\kappa=\kappa(\beta)$ will be positive if $\beta \in(0,1)$ and either $\gamma \geq 1$ or $0<\beta \leq \gamma<1$. The left inequality (A.1) and the right one if $\gamma \geq 1$ have been proved in Lemmas 2.2.4 and 2.2.6 in [21], where $C=1 / \Gamma(\gamma)<1$ and $\kappa(\beta)=\beta^{(\beta /(1-\beta))}(1-\beta)$.

Let us get the right estimate in the case of $0<\beta \leq$ $\gamma<1$. To this end, we use the well-known Wright formula (see e.g., (2.2.5) in [21] or [38]) and represent the function $W(-z ;-\beta, \gamma)$ as

$$
\begin{aligned}
& W(-z ;-\beta, \gamma) \\
& \quad=\gamma W(-z ;-\beta, \gamma+1)+\beta z W(-z ;-\beta, 1+\gamma-\beta) .
\end{aligned}
$$

As we consider the case of $\gamma-\beta \geq 0$ and $\gamma>0$, which means

$$
1+\gamma>1, \quad 1+\gamma-\beta \geq 1
$$

we can apply inequality (A.1) to the terms in the right hand side of (A.2) and get

$$
\begin{aligned}
W(-z ;-\beta, \gamma) \leq & \frac{\exp \left(-\beta^{(\beta /(1-\beta))}(1-\beta) z^{(1 /(1-\beta))}\right)}{\Gamma(\gamma)} \\
& +\frac{\beta z \exp \left(-\beta^{(\beta /(1-\beta))}(1-\beta) z^{(1 /(1-\beta))}\right)}{\Gamma(1+\gamma-\beta)} .
\end{aligned}
$$

It is easy to check that $1 / \Gamma(\gamma), \beta / \Gamma(1+\gamma-\beta) \leq 1$ if $0<\beta \leq$ $\gamma<1$ and that

$$
\begin{aligned}
& z \exp \left(-\beta^{(\beta /(1-\beta))}(1-\beta) z^{(1 /(1-\beta))}\right) \\
& \leq \begin{cases}\exp \left(-\beta^{(\beta /(1-\beta))}(1-\beta) z^{(1 /(1-\beta))}\right), & 0 \leq z \leq 1 ; \\
\exp \left(-\frac{\beta^{(\beta /(1-\beta))}(1-\beta)}{2} z^{(1 /(1-\beta))}\right), & z>1 .\end{cases}
\end{aligned}
$$

Thus, the last inequalities together with (A.4) allow us to get (A.1) with $C=2$ and $\kappa=\beta^{(\beta /(1-\beta))}(1-\beta) / 2>0$ if $0<\beta \leq$ $\gamma<1$. Note that to prove (i) and the first inequality in (iii) of Lemma 6, we need (A.1) in the case of $0<\beta \leq \gamma<1$.

To prove equality (36), we use formula (2.2.5) from [21] as follows:

$$
\frac{d^{n}}{d t^{n}}\left(t^{\gamma-1} W\left(c t^{-\beta} ;-\beta, \gamma\right)\right)=t^{\gamma-1-n} W\left(c t^{-\beta} ;-\beta, \gamma-n\right),
$$

and represent the function $G(y, t)$ as

$$
G(x, t)=\frac{\partial}{\partial t} W\left(-x t^{-\alpha / 2} a_{0}^{-1 / 2} ;-\frac{\alpha}{2}, 1\right) .
$$

After that, equality (A.7) and estimates (A.1) lead to

$$
\int_{0}^{+\infty} G(x, t) d t=W\left(0 ;-\frac{\alpha}{2}, 1\right) .
$$

Then, using definition (34) of the function $W(z ;-\beta, \gamma)$, we can deduce equality (36). To get inequalities (37), we need the well-known formula to a differentiation of the Wright function (see, e.g. (2.2.22) in [21] or [38]) as follows:

$$
\frac{d}{d z} W(z ;-\beta, \gamma)=W(z ;-\beta, \gamma-\beta) .
$$

Due to this formula and (A.6), we can represent $\partial G / \partial x, \partial G / \partial t$ as

$$
\begin{gathered}
\frac{\partial G}{\partial x}=-\frac{t^{-1-\alpha / 2}}{a_{0}^{1 / 2}} W\left(-\frac{x t^{-\alpha / 2}}{a_{0}^{1 / 2}} ;-\frac{\alpha}{2},-\frac{\alpha}{2}\right) ; \\
\frac{\partial G}{\partial t}=t^{-2} W\left(-\frac{x t^{-\alpha / 2}}{a_{0}^{1 / 2}} ;-\frac{\alpha}{2},-1\right) .
\end{gathered}
$$


Furthermore, using inequalities (16) and (17) from Lemma 3 [22], we infer the estimate of the Wright function

$$
|W(-z ;-\beta, \gamma)| \leq \frac{\text { const. }}{1+|z|^{-((\gamma-1) / \beta)}}, \quad \text { if } \gamma<1 .
$$

Thus, representations (A.10) and inequality (A.11) lead to (37). Note that statements (iv)-(vii) of Lemma 6 follow, after some simple calculations, from representations (A.10) and estimate (A.11). [41]:

In virtue of formulas (2.2.4) in [21] or (11) and (14) in

$$
\begin{array}{r}
D_{t}^{\nu}\left(t^{\gamma-1} W\left(-c t^{-\beta} ;-\beta, \gamma\right)\right)=t^{\gamma-\gamma-1} W\left(-c t^{-\beta} ;-\beta, \gamma-\nu\right), \\
I_{t}^{v}\left(t^{\gamma-1} W\left(-c t^{-\beta} ;-\beta, \gamma\right)\right)=t^{\gamma+\nu-1} W\left(-c t^{-\beta} ;-\beta, \gamma+\nu\right), \\
\text { if } \operatorname{Re} \nu>0,
\end{array}
$$

and equality (A.9), we state (42).

\section{B. The proof of Lemma 10}

Using estimate of Lemma 3.1 in [26] or Theorem 1.6 in [29]:

$$
\left|E_{\gamma, \beta}(z)\right| \leq \frac{C}{1+|z|}, \quad \mu \leq|\arg z| \leq \pi,
$$

we have got

$$
\left|E_{\alpha / 2, \alpha}\left(-b_{0} a_{0}^{-1 / 2} t^{\alpha / 2}\right)\right| \leq \frac{C}{1+t^{\alpha / 2}}, \quad \mu=\frac{2 \pi \alpha}{3} .
$$

Then

$$
\left|G_{2}(t)\right| \leq \frac{C t^{\alpha-1}}{1+t^{\alpha / 2}}
$$

Due to formula (E.32) in [42], we can conclude that $G_{2}(t)$ is positive for $t>0$. Thus, the statement (i) of Lemma 10 follows immediately from (B.3) and the arguments above.

Due to formula (1.82) in [29]:

$$
\begin{gathered}
I_{t}^{\nu}\left(t^{\beta-1} E_{\gamma, \beta}\left(\lambda t^{\gamma}\right)\right)=t^{\beta+\nu-1} E_{\gamma, \beta+\nu}\left(\lambda t^{\gamma}\right), \quad \operatorname{Re} \nu>0, \\
D_{t}^{\nu}\left(t^{\beta-1} E_{\gamma, \beta}\left(\lambda t^{\gamma}\right)\right)=t^{\beta-\nu-1} E_{\gamma, \beta-\nu}\left(\lambda t^{\gamma}\right),
\end{gathered}
$$

we can calculate value of $I_{t}^{1-\alpha} G_{2}(t)$ and get

$$
I_{t}^{1-\alpha} G_{2}(t)=E_{\alpha / 2,1}\left(-b_{0} a_{0}^{-1 / 2} t^{\alpha / 2}\right)=\sum_{k=0}^{+\infty} \frac{\left(-b_{0} a_{0}^{-1 / 2} t^{\alpha / 2}\right)^{k}}{\Gamma(1+(\alpha k / 2))} \text {. }
$$

Here we used the definition of $E_{\gamma, \beta}(z)$ (see (89)). Then, representation (B.6) leads to (91). Note that $D_{t}^{\alpha} G_{2}(t)=$ $(d / d t) I_{t}^{1-\alpha} G_{2}(t)$. Hence

$$
\begin{aligned}
\int_{0}^{+\infty} & D_{\tau}^{\alpha} G_{2}(\tau) d \tau \\
& =\int_{0}^{+\infty} \frac{d}{d \tau} I_{\tau}^{1-\alpha} G_{2}(\tau) d \tau \\
& =\lim _{\tau \rightarrow+\infty} I_{\tau}^{1-\alpha} G_{2}(\tau)-\lim _{\tau \rightarrow 0} I_{\tau}^{1-\alpha} G_{2}(\tau),
\end{aligned}
$$

or due to (B.6) and (91),

$$
\int_{0}^{+\infty} D_{\tau}^{\alpha} G_{2}(\tau) d \tau=\lim _{\tau \rightarrow+\infty} E_{\alpha / 2,1}\left(-b_{0} a_{0}^{-1 / 2} \tau^{\alpha / 2}\right)-1 .
$$

To obtain (92), we apply estimate like (B.1) to the first term in the right-hand side (B.8). In the same way, we infer inequality (95).

Inequalities (93)-(95) follow from (B.5)-(B.8) and (B.1).

At last, proving inequality (96) is enough to use formula (1.83) in [29] as follows:

$$
\frac{d}{d t}\left(t^{\beta-1} E_{\gamma, \beta}\left(t^{\gamma}\right)\right)=t^{\beta-2} E_{\gamma, \beta-1}\left(t^{\gamma}\right),
$$

and to estimate (B.1).

\section{Acknowledgments}

The authors are very grateful to the referee for the careful reading of the paper and the valuable comments. This work is partially supported by Grant PIRSES-GA-2011-295164.

\section{References}

[1] A. A. Kilbas, H. M. Srivastava, and J. J. Trujillo, North-Holland Mathematics Studies, vol. 204 of Theory and Applications of Fractional Differential Equations, Elsevier Science, Amsterdam, The Netherlands, 2006.

[2] A. Friedman and M. Shinbort, "The initial value problem for the linearized equation of water-waves," Journal of Mathematics and Mechanics, vol. 17, pp. 107-180, 1967.

[3] H. S. Carslaw and J. C. Jager, Conduction of Heat in Solids, Oxford University Press, Oxford, UK, 1959.

[4] B. V. Bazaliy, "On the Stefan problem," Doklady Akademii Nauk Ukrainy, vol. 11, pp. 3-7, 1986.

[5] V. V. Barkovskii and V. A. Kul'chitskii, On Week Solutions of Some Mixed Problems for the Schrödinger, Linear and Nonlinear Boundary Problems, Kyiv, Ukraina, 1971.

[6] V. A. Kul'chitskii, Mathematical Model and Qualitative Investigation of the Induction Heating, Nonlinear Boundary Problems of Mathematical Physics, Kyiv, Ukraina, 1973.

[7] M. I. Khazan, "On boundary-value problems with evolutional boundary condition," Linear and Nonlinear Boundary Problems, vol. 10, pp. 105-115, 1986.

[8] N. A. Grigor'eva and M. S. Mogilevskii, On a Boundary Value Problem for the Heat Equation, Geometrical Problems in the Theory of Functions and Sets, Kalinin, 1985.

[9] G. I. Bizhanova and V. A. Solonnikov, "On the solvability of an initial-boundarty value problem for a second-order parabolic equation with a time derivative in the boundary condition in a weighted Hölder space of functions," St. Petersburg Mathematical Journal, vol. 5, pp. 97-124, 1994.

[10] J. Escher, "Quasilinear parabolic systems with dynamical boundary conditions," Communications in Partial Differential Equations, vol. 18, pp. 1309-1364, 1993.

[11] M. Fila and P. Quittner, "Large time behavior of solutions of a semilinear parabolic equation with a nonlinear dynamical boundary condition," in Topics in Nonlinear Analysis, vol. 35 of Progress in Nonlinear Differential Equations and Their Applications, Birkhäuser, Basel, Switzerland, 1999. 
[12] E. Vitillaro, "Global existence for the heat equation with nonlinear dynamical boundary conditions," Royal Society of Edinburgh A, vol. 135, no. 1, pp. 175-207, 2005.

[13] J. Bouchaud and A. Georges, "Anomalous diffusion in disordered media: Statistical mechanisms, models and physical applications," Physics Report, vol. 195, no. 4-5, pp. 127-293, 1990.

[14] R. Metzler and J. Klafter, "Boundary value problems for fractional diffusion equations," Physica A, vol. 278, no. 1, pp. 107-125, 2000.

[15] G. Drazer and D. H. Zanette, "Experimental evidence of powerlaw trapping-time distributions in porous media," Physical Review E, vol. 60, no. 5B, pp. 5858-5864, 1999.

[16] E. R. Weeks and D. A. Weitz, "Subdiffusion and the cage effect studied near the colloidal glass transition," Chemical Physics, vol. 284, no. 1-2, pp. 361-367, 2002.

[17] K. Ritchie, X. Shan, J. Kondo, K. Iwasawa, T. Fujiwara, and A. Kusumi, "Detection of non-Brownian diffusion in the cell membrane in single molecule tracking," Biophysical Journal, vol. 88, no. 3, pp. 2266-2277, 2005.

[18] J. Klafter, G. Zumofen, and M. F. Shlesinger, in ThePhysics of Complex Systems, F. Mallamace and H. E. Stanley, Eds., IOS Press, Amsterdam, The Netherlands, 1997.

[19] A. N. Kochubei, "A Cauchy problem for evolution equations of fractional order," Journal of Differential Equations, vol. 25, pp. 967-974, 1989.

[20] A. N. Kochubei, "Fractional order diffusion," Journal of Differential Equations, vol. 26, pp. 485-492, 1990.

[21] A. V. Pskhu, Partial Differential Equations of the Fractional Order, Nauka, Moscow, Russia, 2005, (Russian).

[22] A. V. Pskhu, "The fundamental solution of a diffusion-wave equation of fractional order," Izvestiya Mathematics, vol. 73, no. 2, pp. 351-392, 2009.

[23] V. D. Gejji and H. Jafari, "Boundary value problems for fractional diffusion-wave equation," The Australian Journal of Mathematical Analysis and Applications, vol. 3, pp. 1-8, 2006.

[24] O. P. Agrawal, "Solution for a fractional diffusion-wave equation defined in a bounded domain," Nonlinear Dynamics, vol. 29, no. 1-4, pp. 145-155, 2002.

[25] G. M. Mophou and G. M. N’Guérékata, "On a class of fractional differential equations in a Sobolev space," Applicable Analysis, vol. 91, pp. 15-34, 2012.

[26] K. Sakamoto and M. Yamamoto, "Initial value/boundary value problems for fractional diffusion-wave equations and applications to some inverse problems," Journal of Mathematical Analysis and Applications, vol. 382, no. 1, pp. 426-447, 2011.

[27] S. G. Samko, A. A. Kilbas, and O. I. Marichev, Fractional Integrals and Derivatives, Gordon and Breach Science, Philadelphia, Pa, USA, 1993.

[28] F. Mainardi, "Fractional calculus: some basic problems in continuum and statistical mechanics," in Fractals and Fractional Calculus in Continuum Mechanics, A. Garpinteri and F. Mainardi, Eds., pp. 291-348, Springer, New York, NY, USA, 1997.

[29] I. Podlubny, Fractional Differential Equations, Academic Press, San Diego, Calif, USA, 1999.

[30] P. Clément, S. Londen, and G. Simonett, "Quasilinear evolutionary equations and continuous interpolation spaces," Journal of Differential Equations, vol. 196, no. 2, pp. 418-447, 2004.

[31] M. Kirane and N. Tatar, "Absence of local and global solutions to an elliptic system with time-fractional dynamical boundary conditions," Siberian Mathematical Journal, vol. 48, no. 3, pp. 477-488, 2007.
[32] O. A. Ladyzhenskaia, V. A. Solonnikov, and N. N. Ural'tseva, Linear and Quasilinear Parabolic Equations, Academic Press, New York, NY, USA, 1968.

[33] F. Huang and F. Liu, "The space-time fractional diffusion equation with Caputo derivatives," Journal of Applied Mathematics and Computing, vol. 19, no. 1-2, pp. 179-190, 2005.

[34] I. Podlubny, "The Laplace transformation for linear differential equation of the fractional order," http://arxiv.org/abs/funct-an/9710005.

[35] A. Erdelyi, Higher Transcendental Functions V.1,3, McGraw-Hill, New York, NY, USA, 1955.

[36] A. Kilbas, "Fractional calculus of the generalized Wright functions," Fractional Calculus and Applied Analysis, vol. 8, pp. 113126, 2005.

[37] N. V. Krylow, Lectures on Elliptic and Parabolic Equations in Hölder Spaces, AMS, 1996.

[38] E. M. Wright, "The generalized bessel function of order greater than one," Quarterly Journal of Mathematics, vol. 11, no. 1, pp. 36-48, 1940.

[39] M. Krasnoschok and N. Vasylyeva, "On a solvability of a nonlinear fractional reaction-diffusion system in the Hölder spaces," Journal of Nonlinear Studies. In press.

[40] R. P. Agarwal, "A propos d'une note de M. Pierre Humbert," Comptes Rendus Seances de l'Académie des Sciences, vol. 236, no. 21, pp. 2031-2032, 1953.

[41] A. N. Kochubei, "Fractional-parabolic systems," Potential Analysis, vol. 37, no. 1, pp. 1-30, 2012.

[42] F. Mainardi, Fractional Calculus and Waves in Linear Viscoelasticity, Imperial College Press, London, UK, 2010. 


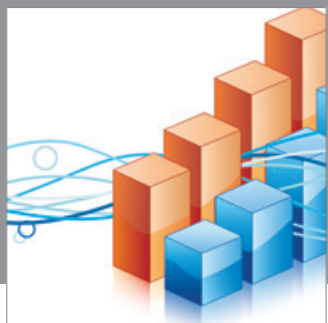

Advances in

Operations Research

mansans

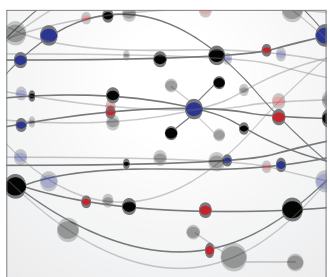

The Scientific World Journal
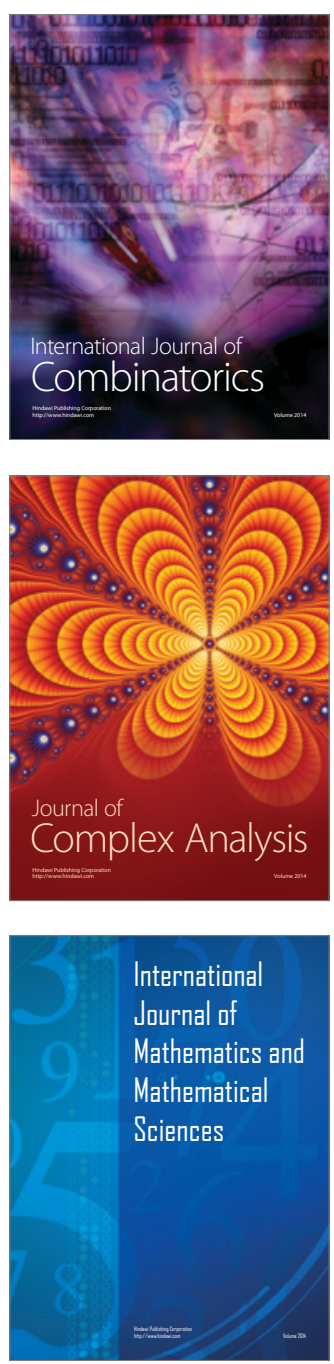
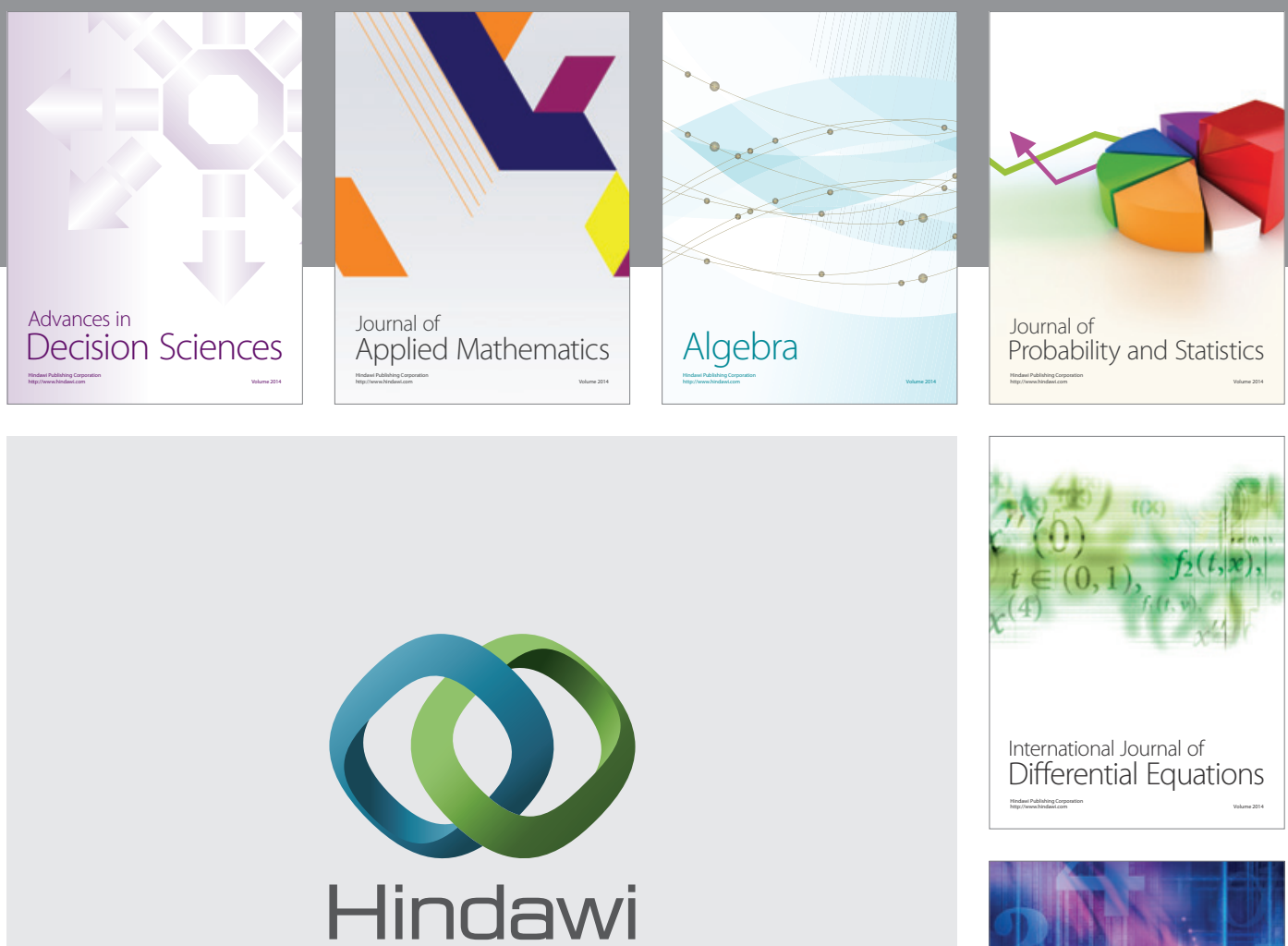

Submit your manuscripts at http://www.hindawi.com
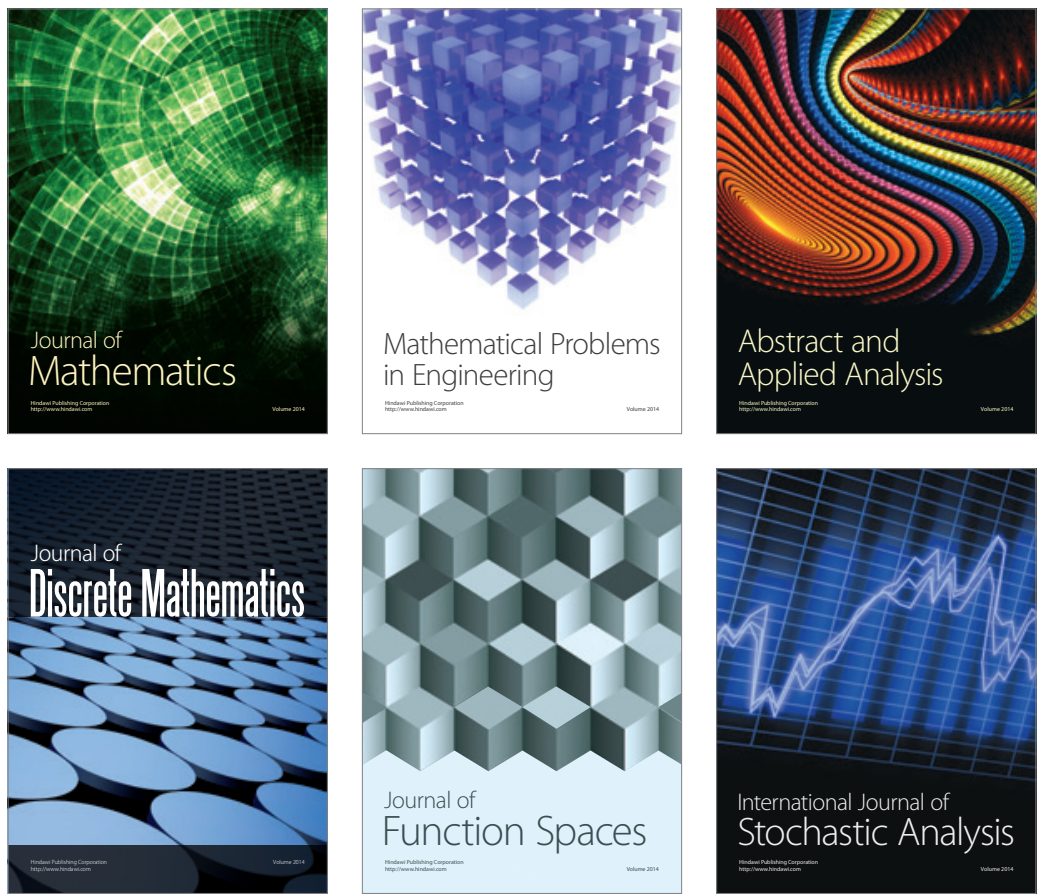

Journal of

Function Spaces

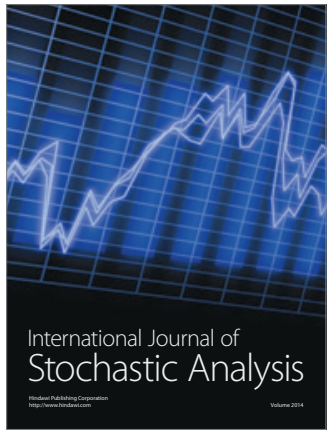

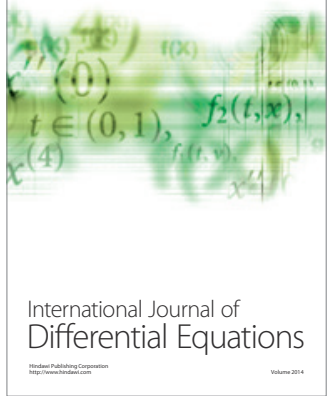
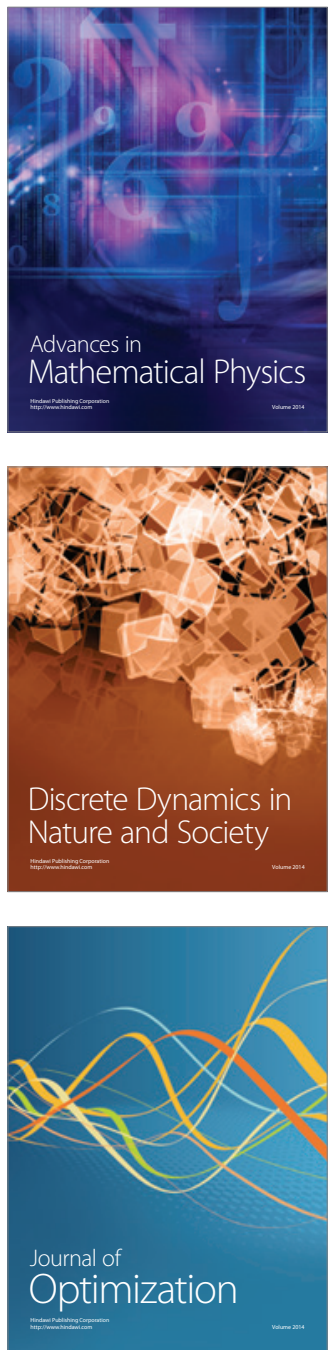